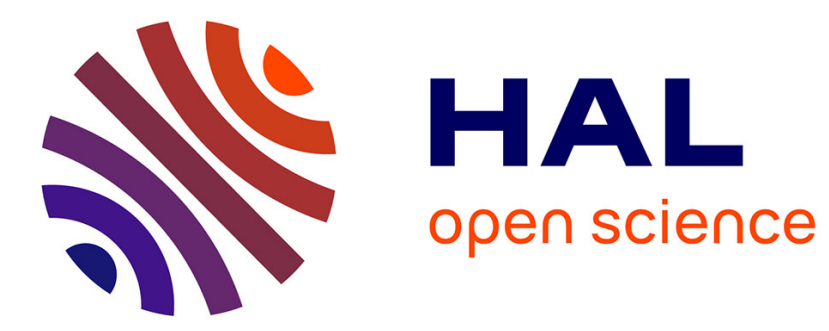

\title{
Performance Study of a Near Maximum Likelihood Code-Aided Timing Recovery Technique
}

\author{
Imen Nasr, Benoit Geller, Leila Najjar Atallah, Sofiane Cherif
}

\section{To cite this version:}

Imen Nasr, Benoit Geller, Leila Najjar Atallah, Sofiane Cherif. Performance Study of a Near Maximum Likelihood Code-Aided Timing Recovery Technique. IEEE Transactions on Signal Processing, 2016, 10.1109/TSP.2015.2489602 . hal-01248388

HAL Id: hal-01248388

https://hal-ensta-paris.archives-ouvertes.fr/hal-01248388

Submitted on 25 Dec 2015

HAL is a multi-disciplinary open access archive for the deposit and dissemination of scientific research documents, whether they are published or not. The documents may come from teaching and research institutions in France or abroad, or from public or private research centers.
L'archive ouverte pluridisciplinaire HAL, est destinée au dépôt et à la diffusion de documents scientifiques de niveau recherche, publiés ou non, émanant des établissements d'enseignement et de recherche français ou étrangers, des laboratoires publics ou privés. 


\title{
Performance Study of a Near Maximum Likelihood Code-Aided Timing Recovery Technique
}

\author{
Imen Nasr ${ }^{1,2}$, Benoît Geller ${ }^{2}$, Leïla Najjar Atallah ${ }^{1}$ and Sofiane Cherif ${ }^{1}$ \\ ${ }^{1}$ COSIM Research Lab, Higher School of Communications of Tunis (SUP'COM), University of Carthage, Tunisia \\ ${ }^{2}$ U2IS Research Lab, Ecole Nationale Supérieure de Techniques Avancées ENSTA ParisTech, UPSA, France \\ 1 \{nasr.imen, leila.najjar, sofiane.cherif\}@ supcom.rnu.tn \\ ${ }_{2}\{$ imen.nasr, benoit.geller $\} @$ ensta-paristech.fr
}

\begin{abstract}
In this paper, we propose a new code-aided (CA) timing recovery algorithm for various linear constant modulus constellations based on the Maximum Likelihood (ML) estimator. The first contribution is the derivation of a soft estimator expression of the transmitted symbol instead of its true or hard estimated value which is fed into the timing error detector (TED) equation. The proposed expression includes the Log-Likelihood Ratios (LLRs) obtained from a turbo decoder. Our results show that the proposed $\mathrm{CA}$ approach achieves almost as good results as the data-aided (DA) approach over a large interval of SNR values while achieving a higher spectral efficiency. We also derive the corresponding CA Cramer Rao Bounds (CRB) for various modulation orders. Contrarily to former work, we develop here the CRB analytical expression for different $M$-PSK modulation orders and validate them through comparison to empirical CRB obtained by Monte Carlo iterations. The proposed CA estimator realizes an important gain over the non data-aided approach (NDA) and achieves a smaller gap when compared to its relative CA CRB, especially at moderate SNR values where modern systems are constrained to work.
\end{abstract}

\section{INTRODUCTION}

Synchronization is among the most important tasks performed in a communication system receiver prior to detection and decoding. The main issue in a synchronization scheme is to work properly even at very low SNR and this is a hard task as it is processed at the front end. In this paper, we focus on time delay synchronization for linear constant modulus modulation systems.

Timing recovery is required to ensure that the sampling instants coincide with the maximum opening of the signal eye-diagram thus maximizing the robustness to noise and interference. Some DA and non-data-aided (NDA) time delay estimation techniques have been employed in real systems for decades such as those reported in [1] and [2]. However, in a DA mode, we need to insert some pilot signals which leads to the loss of throughput and the decrease of spectrum efficiency. In the NDA mode, no reference signals are needed and time synchronization is performed using only the received signal. As a result, the system performance can be deteriorated due to the loss of some statistical information about the transmitted signal especially in harsh channel conditions. Nevertheless, with the development of channel coding techniques [3]-[7],

\footnotetext{
* This work has been supported by the Greencocom project of the ANR program
}

more researches have focused on CA synchronization algorithms [8] which consist in taking advantage of the decoding gain to improve the estimation performance of the desired parameters. The frame of iterative receivers can help the synchronizer to better estimate the time delay by exploiting the soft information about the received symbols. This technique has already been used for phase estimation in turbo decoding receivers [9]. It was also exploited in [10] with an expectation maximization algorithm in the Maximum Likelihood (ML) synchronization framework. Moreover, the timing synchronizer and the channel decoder can improve each other progressively by exchanging information using iterative techniques. Reference [11] presents a maximum likelihood timing estimator which uses the extrinsic information generated from the decoder to perform time synchronization for BPSK signals. In [12] the authors have proposed to introduce the timing recovery inside the turbo equalizer to jointly perform these tasks. Unfortunately, this technique requires a large number of turbo iterations, especially when the timing jitter is large. In [13], an iterative timing recovery technique where the soft information from the decoder is fed into the Mueller and Muller (M\&M) Timing Error Detector (TED) [1] is jointly performed with turbo equalization for BPSK signals. We have already proposed a soft maximum likelihood timing recovery technique for BPSK signals in [14] and for square QAM signals in [15] and this soft estimation is valid for any kind of timing detector.

The first contribution of this paper is to propose a new soft time delay estimation for $M$-PSK modulated signals. This expression is derived from the likelihood function conditioned to the time delay and used by the ML timing error detector instead of the effective or hard estimated symbol. The proposed expression includes the LLRs obtained from a soft decoder such as a turbo decoder.

Although many estimators have been proposed so far, theoretically assessing their relevance compared to the best achievable performance is also of a great interest. Thus, we have to resort to compare the proximity of both analytical and simulation results to some lower bounds corresponding to the optimum performance. For instance, lower bounds on the MSE give an indication of the performance limitations, and consequently determine whether the performance requirements in terms of MSE are realistic or not and if enhancements are still possible. Although there exists many families of lower 
bounds, the Cramer-Rao bound (CRB) family is the most commonly used and the easiest to determine [16]. There are several works concerning the CA CRBs for carrier phase and frequency estimation. In [17]-[19], the authors derive the Bayesian and hybrid Cramer-Rao bounds (BCRB and HCRB) for the CA, the DA, and the NDA dynamical phase estimation of linearly modulated signals and theoretically show the possible improvement brought by a soft CA technique. In reference [20], a forward-backward algorithm was proposed to cope with the performance deterioration in the NDA mode; although this technique has sometimes shown better performance than the CA approach, it can only be implemented by an off-line manner. A comparison between different timing techniques in terms of BER has been proposed in [21] using the extrinsic information transfer chart (EXIT chart) [22]. Nevertheless, some statistical properties of the estimators such as the bias and the mean square error (MSE) cannot be provided by the EXIT chart analysis. The authors of [23] have derived semianalytical expressions of the estimator mean and variance, as function of the timing offset, for a CA decision-directed (DD) timing synchronizer based on M\&M Detector (MMD). However, the performance evaluation has been made only for BPSK signals at low SNR regime, based on the assumption that inter-symbol interference (ISI) could be approximated by an additive Gaussian noise as in [24]. For time recovery, only closed forms of the DA CRB [25] and the NDA CRB for BPSK, MSK and Square-QAM Transmissions [26] have been derived. Differently from [25] and [26], our goal and contribution through this work is also to give closed form expressions of the CRB for CA timing recovery for BPSK, QPSK and in general $M$-PSK modulated signals and to compare the performance of the proposed CA estimator to this bound.

This paper is organized as follows. In section II, the system model and the Decision Directed (DD) time delay estimation based on the ML approach are recalled. In section III, the derivation of various CA time delay recovery techniques is proposed. Then, in section IV closed form expressions of some CRBs for different types of linear modulations are derived. Simulation results are provided in section $\mathrm{V}$ and validate our analysis. The last section concludes our work.

\section{GLOSSARY OF PRINCIPAL NOTATIONS}

- $\Re\{z\}$ and $\Im\{z\}$ : the real and imaginary parts of $z$

- $z^{*}$ : the conjugate of $z$

- $y_{k, 1}$ : the real part of any variable $y_{k}$

- $y_{k, 2}$ : the imaginary part of any variable $y_{k}$

- $\otimes:$ the convolution operation

- $f^{\prime}$ (resp. $\left.f^{\prime \prime}\right)$ : the first (resp. the second) derivative of $f$, for any function $f$

- $f_{X}(x)$ : the probability density function (pdf) of the random variable $X$

- $P(X)$ : the probability of the random variable $X$

- $E(X)$ : the mean value of the random variable $X$

\section{Decision Directed Time Delay Estimation}

Let us consider the linearly modulated transmitted signal $s(t)$ written as:

$$
s(t)=\sum_{i} a_{i} h(t-i T),
$$

where $a_{i}$ denotes the zero mean i.i.d. transmitted symbols drawn from a given finite size constellation, $h(t)$ is the impulse response of the transmission filter and $T$ is the symbol period.

The received signal is:

$$
r(t)=s(t-\tau)+n(t),
$$

where the channel introduces an unknown delay $\tau$ to the transmitted signal $s(t)$. In (2) the received signal is disturbed by an additive white Gaussian noise (AWGN) $n(t)$ of zero mean and of variance $\sigma^{2}$.

The time delay is estimated in the maximum likelihood sense by maximizing the likelihood function according to the following equation [27]:

$$
\hat{\tau}=\arg \max _{u} \Lambda(u, \mathbf{a}),
$$

where $\mathbf{a}$ is the vector of the transmitted symbols,

$$
\Lambda(u, \mathbf{a})=\exp \left(-\frac{1}{2 \sigma^{2}} \int_{T_{0}}|r(t)-s(t-u)|^{2} d t\right),
$$

is the likelihood function and $T_{0}$ is the observation interval. Equivalently, the log-likelihood function $\Lambda_{L}(u, \mathbf{a})$ can be used instead of $\Lambda(u, \mathbf{a})$. According to [28], when $T_{0}$ is large enough, from (1)-(4) we estimate the time delay in the maximum likelihood sense according to:

$$
\hat{\tau}=\arg \max _{u} \Lambda_{L}(u, \mathbf{a}),
$$

where:

$$
\Lambda(u, \mathbf{a})=\prod_{j} \exp \left(\frac{\Re\left\{a_{j}^{*} x_{j}(u)\right\}}{\sigma^{2}}\right),
$$

or equivalently:

$$
\Lambda_{L}(u, \mathbf{a})=\log (\Lambda(u, \mathbf{a}))=\sum_{j} \frac{\Re\left\{a_{j}^{*} x_{j}(u)\right\}}{\sigma^{2}},
$$

and:

$$
\begin{aligned}
x_{j}(u) & =y_{j}(u)+v_{j}(u), \\
y_{j}(u) & =\sum_{i} a_{i} g((j-i) T-(\tau-u)), \\
v_{j}(u) & =\int_{T_{0}} h^{*}(t-j T-u) n(t) d t, \\
g(t) & =h \otimes h_{-}^{*}(t),
\end{aligned}
$$

where $h_{-}(t)=h(-t)$, for any function $h . x_{j}(u)$ is seen as the matched filter output of the received signal, $y_{j}(u)$ is the useful part, $v_{j}(u)$ is a colored gaussian noise of zero mean and variance $\sigma^{2}$ and $g$ is a Nyquist pulse.

Since it is difficult in practice to maximize analytically (7) with respect to $u$, adaptive algorithms are implemented whose 
objective is to minimize the instantaneous error at each time instant $k$ using the following equation:

$$
\tau_{k}=\tau_{k-1}+\mu e_{k}\left(a_{k}, \tau_{k-1}\right),
$$

where $\tau_{k}$ is the estimate of $\tau$ at time index $k, \mu$ is the step size and $e_{k}\left(a_{k}, \hat{\tau}_{k-1}\right)$ is the updating error term expressed as:

$$
e_{k}\left(a_{k}, \tau_{k-1}\right)=\Re\left\{\left.a_{k}^{*} \frac{\partial x_{k}(\tau)}{\partial \tau}\right|_{\tau=\tau_{k-1}}\right\} .
$$

Such approach is called the ML-based Detector (MLD) by the authors of [29]. The term $\left.\frac{\partial x_{k}(\tau)}{\partial \tau}\right|_{\tau=\tau_{k-1}}$ can be obtained by first interpolating the sampled version of the received signal using a polynomial interpolator, then differentiating the interpolated polynomial function with respect to $\tau$ and finally evaluating the derivative at $\tau_{k-1}$. However, the proposed computation method of $e_{k}\left(a_{k}, \tau_{k-1}\right)$ leads to high implementation complexity because of the presence of the derivative of the matched filter denoted $h_{-}^{\prime}(t)$.

Other expressions of the updating term in the adaptive estimation of $\tau$ have been proposed in the literature by approximating the derivative of $x_{k}(\tau)$ using a finite difference method. Table I summarizes the updating term expression of the most known Timing Error Detectors (TED) which are the M\&M Detector (MMD) [1], the Zero Crossing Detector (ZCD) [30] and the Early Late Detector (ELD) [31].

TABLE I: Timing Detectors

\begin{tabular}{|l|l|}
\hline TED & updating term: $e_{k}\left(a_{k}, \tau_{k-1}\right)$ \\
\hline MMD & $a_{k} x_{k-1}\left(\tau_{k-1}\right)-a_{k-1} x_{k}\left(\tau_{k-1}\right)$ \\
\hline ZCD & $x_{k-\frac{1}{2}}\left(\tau_{k-1}\right)\left[a_{k}-a_{k-1}\right]$ \\
\hline ELD & $a_{k}\left[x_{k+\frac{1}{2}}\left(\tau_{k-1}\right)-x_{k-\frac{1}{2}}\left(\tau_{k-1}\right)\right]$ \\
\hline
\end{tabular}

The symbols $a_{k}$ which appear in the updating term can be known if some pilot symbols are sent within the data frame. In this case, the TED is operating in a DA mode. In order to enhance the spectral efficiency, these pilot symbols can be omitted and $a_{k}$ are estimated at the receiver. The TED is then operating in a DD mode. To do so, we usually implement a NDA mode in which a hard estimation of the symbols $a_{k}$ is used. Nevertheless, this technique performance degrades rapidly at low SNR, where modern systems are constrained to work. Departing from the classical ML approach and the previous simplified proposed versions, we hereafter propose a new timing detector using soft estimated symbols instead of known $a_{k}$ or DD hard estimation of $a_{k}$, in the updating term. The new time delay estimation technique takes advantage of the soft information from the decoder block to ameliorate the timing recovery results. The proposed algorithm derivation and performance evaluation are going to be presented in the next sections.

\section{Proposed Code Aided Timing Detector}

Instead of making possibly unreliable hard decisions on the received data (NDA approach) or reducing spectral efficiency by sending preamble sequences (DA Approach), we propose to take advantage of the system decoder output, in order to enhance the time recovery performance. In the following paragraphs, we derive a code aided adaptive algorithm based on the maximum likelihood approach for $M$-PSK signals.

We assume the constellation to be Gray-coded taking values in the alphabet set $\mathcal{V}=\left\{v_{0}, v_{1}, \ldots, v_{M-1}\right\}$ where $M$ is the constellation size. We suggest the following notation for the $i^{\text {th }}$ Gray-coded constellation element $v_{i}$ and the $k^{\text {th }}$ transmitted symbol $a_{k}$ :

$$
\begin{aligned}
v_{i} & \Leftrightarrow c_{1}^{i} c_{2}^{i} \ldots c_{\log _{2}(M)}^{i}, \\
a_{k} & \Leftrightarrow b_{1}^{k} b_{2}^{k} \ldots b_{\log _{2}(M)}^{k},
\end{aligned}
$$

where $c_{j}^{i}$ (resp. $b_{j}^{k}$ ) corresponds to the $i^{t h}$ binary information of $v_{i}$ (resp. $\left.a_{k}\right)$.

By choosing a large-size interleaver, we can assume that coded bits in a symbol are statistically independent [4] [32] so that the a priori probability of the transmitted symbol $a_{k}$ to be equal to a possible symbol $v_{i}$ of the alphabet is:

$$
\begin{aligned}
P\left[a_{k}=v_{i}\right] & =P\left[b_{1}^{k}=c_{1}^{i}, b_{2}^{k}=c_{2}^{i}, \ldots, b_{\log _{2}(M)}^{k}=c_{\log _{2}(M)}^{i}\right] \\
& =\prod_{m=1}^{\log _{2}(M)} P\left[b_{m}^{k}=c_{m}^{i}\right] .
\end{aligned}
$$

Let us consider, $\lambda_{m}^{k}=\log \left(\frac{P\left[b_{m}^{k}=1\right]}{P\left[b_{m}^{k}=0\right]}\right)$ the soft output of the decoder at any time index $k$. Assuming that the symbol $v_{i}$ is transmitted at time instant $k$, a general formula of the a priori probability of the coded bit $b_{m}^{k}$, using $P\left[b_{m}^{k}=0\right]+P\left[b_{m}^{k}=1\right]=1$, is given by:

$$
P\left[b_{m}^{k}=c_{m}^{i}\right]=\frac{\exp \left(\left(2 c_{m}^{i}-1\right) \frac{\lambda_{m}^{k}}{2}\right)}{2 \cosh \left(\frac{\lambda_{m}^{k}}{2}\right)} .
$$

The soft information $\lambda_{m}^{k}$ can either be obtained simply from a soft demapper or, after several decoding iterations, by a soft decoder. In practice, it is easier to process soft demapper data rather than soft decoder values. At low SNR however, the coding gain is advantageous especially for high order constellations leading the soft decoding a more interesting approach.

Unlike (3) and (5) and in the absence of training sequences, we consider the ML estimator averaged over all possible realizations:

$$
\hat{\tau}=\arg \max _{u} \Lambda_{L}(u)=\arg \max _{u} \log (\Lambda(u)),
$$

where $\Lambda(u)$ is obtained by averaging the likelihood function $\Lambda(u, \mathbf{a})$ over the possible set of transmitted symbols $a_{k}$.

Thus, averaging (6) over the set of possible transmitted symbols and using their independence property, we obtain:

$$
\Lambda(u)=\prod_{k} \sum_{v_{i} \in \mathcal{V}} P\left[a_{k}=v_{i}\right] \exp \left(\frac{\Re\left\{v_{i}^{*} x_{k}(u)\right\}}{\sigma^{2}}\right)
$$

where $P\left[a_{k}=v_{i}\right]$ is given by (16)-(17).

In the following, we derive separately the CA TED for BPSK, QPSK and $M$-PSK modulated signals. Even if BPSK and QPSK can be considered as 2-PSK and 4-PSK respectively, for CA TED we use a recursive technique that works only for larger $M$-PSK, with constellation size $M \geq 8$. 


\section{A. BPSK modulation}

In this section, we present the code aided adaptive time recovery algorithm with a BPSK modulation which is a subcase of $M$-PSK with $M=2$ and $m=1$. In this case, $v_{i} \in\{-1,1\}$ and $b_{k} \in\{0,1\}$. Using (16) and (17), the Likelihood function in (19) for a BPSK signal becomes:

$$
\Lambda(u)=\prod_{k} \frac{\cosh \left(\frac{\lambda_{k}}{2}+\frac{x_{k, 1}(u)}{\sigma^{2}}\right)}{\cosh \left(\frac{\lambda_{k}}{2}\right)},
$$

where, for simplification, $\lambda_{k}$ denotes $\lambda_{1}^{k}$ and the index 1 in $x_{k, 1}(u)$ refers to the use of the real part of $x_{k}(u)$.

The received signal is corrupted by a real-valued AWGN with power spectral density equal to $\frac{N_{0}}{2}=\sigma^{2}$ so that going back to the log-likelihood function from (20), we obtain the following expression:

$$
\Lambda_{L}(u)=\sum_{k} \ln \left(\frac{\cosh \left(\frac{\lambda_{k}}{2}+\frac{2 x_{k, 1}(u)}{N_{0}}\right)}{\cosh \left(\frac{\lambda_{k}}{2}\right)}\right) .
$$

By differentiating $\Lambda_{L}(u)$ with respect to $u$ we obtain:

$$
\frac{\partial \Lambda_{L}(u)}{\partial u}=\sum_{k} \frac{2}{N_{0}} \frac{\partial x_{k, 1}(u)}{\partial u} \tanh \left(\frac{\lambda_{k}}{2}+\frac{2 x_{k, 1}(u)}{N_{0}}\right) .
$$

Then in order to estimate $\tau$, we propose in practice to implement the ML adaptive equation (12) or the approximations presented in Table I by substituting $a_{k}$ with the soft symbol:

$$
\tilde{a}_{k}(u)=\tanh \left(\frac{\lambda_{k}}{2}+\frac{2 x_{k, 1}(u)}{N_{0}}\right) .
$$

The updating equation then becomes:

$$
\tau_{k}=\tau_{k-1}+\mu e_{k}\left(\tilde{a}_{k}\left(\tau_{k-1}\right), \tau_{k-1}\right),
$$

where the soft symbol is given by (23).

\section{B. QPSK modulation}

With a QPSK modulation, for which $M=4$ and $m \in$ $\{1,2\}$, the constellation set is $\mathcal{V}=\left\{v_{0}, v_{1}, v_{2}, v_{3}\right\}$ where:

$$
v_{1}=-v_{0}, v_{2}=v_{0}^{*}, v_{3}=-v_{0}^{*},
$$

and we choose the corresponding Gray-coded bits:

$$
v_{0} \Leftrightarrow 11, v_{1} \Leftrightarrow 00, v_{2} \Leftrightarrow 01 \text { and } v_{3} \Leftrightarrow 10 .
$$

The likelihood function is then given by:

$$
\Lambda(u)=\prod_{k} \sum_{i=0}^{3} P\left[a_{k}=v_{i}\right] \exp \left(\frac{\Re\left\{v_{i}^{*} x_{k}(u)\right\}}{\sigma^{2}}\right) .
$$

The expression of $P\left[a_{k}=v_{i}\right]$ can be obtained using (16), (17) and (26) as:

$$
P\left[a_{k}=v_{i}\right]=\beta_{k} \exp \left(\frac{\left(2 c_{1}^{i}-1\right) \lambda_{1}^{k}+\left(2 c_{2}^{i}-1\right) \lambda_{2}^{k}}{2}\right),
$$

where $\beta_{k}=\frac{1}{4 \cosh \left(\lambda_{1}^{k} / 2\right) \cosh \left(\lambda_{2}^{k} / 2\right)}$.

By replacing $P\left[a_{k}=v_{i}\right]$ in (27) by its expression (28) and using (25), we get:

$$
\begin{aligned}
\Lambda(u) & =\prod_{k} 2 \beta_{k}\left[\cosh \left(\frac{\lambda_{1}^{k}+\lambda_{2}^{k}}{2}+\frac{\Re\left\{v_{0}^{*} x_{k}(u)\right\}}{\sigma^{2}}\right)\right. \\
& \left.+\cosh \left(\frac{\lambda_{2}^{k}-\lambda_{1}^{k}}{2}+\frac{\Re\left\{v_{0}^{*} x_{k}(u)\right\}}{\sigma^{2}}\right)\right] .
\end{aligned}
$$

Given that $\cosh (a)+\cosh (b)=2 \cosh \left(\frac{a+b}{2}\right) \cosh \left(\frac{a-b}{2}\right)$ and that the received signal is corrupted by a complex-valued AWGN with power spectral density equal to $N_{0}=\sigma^{2}$, we obtain:

$$
\begin{aligned}
\Lambda(u) & =\prod_{k} 4 \beta_{k} \cosh \left(\frac{\lambda_{2}^{k}}{2}+\frac{2 v_{0,1} x_{k, 1}(u)}{N_{0}}\right) \\
& \times \cosh \left(\frac{\lambda_{1}^{k}}{2}+\frac{2 v_{0,2} x_{k, 2}(u)}{N_{0}}\right) .
\end{aligned}
$$

Evaluating and differentiating the log-likelihood function $\Lambda_{L}(u)$ with respect to $u$ leads to:

$$
\frac{\partial \Lambda_{L}(u)}{\partial u}=\frac{2}{N_{0}} \sum_{k} \Re\left\{\tilde{a}_{k}^{*}(u) \frac{\partial x_{k}(u)}{\partial u}\right\},
$$

where:

$$
\begin{aligned}
& \tilde{a}_{k, 1}(u)=v_{0,1} \tanh \left(\frac{\lambda_{2}^{k}}{2}+\frac{2 v_{0,1} x_{k, 1}(u)}{N_{0}}\right), \\
& \tilde{a}_{k, 2}(u)=v_{0,2} \tanh \left(\frac{\lambda_{1}^{k}}{2}+\frac{2 v_{0,2} x_{k, 2}(u)}{N_{0}}\right) .
\end{aligned}
$$

In practice, like for the BPSK case, we propose to estimate $\tau$, using the updating equation (24) where the real and imaginary parts of $\tilde{a}_{k}$ are respectively given by (32) and (33) for $u=\tau_{k-1}$.

\section{M-PSK modulation}

In this paragraph, we derive an adaptive TED algorithm equation in the case of $M$-PSK modulated signals. These derivations are indeed built based on the Gray coding properties and exploit the relationship between constellations of order $p$ and $p-2$ which makes the analysis valid only for $p \geq 3$. All the analytical expressions are valid for signals that are mapped from 3-bits and more. Stated differently, the above results related to BPSK and QPSK signals cannot be derived as particular cases.

In fact, an $M$-PSK modulated signal takes values from the set $\mathcal{V}_{2^{p}}=\left\{v_{m}=e^{\frac{j 2 \pi m}{M}}, m=1, \ldots, M\right\}$, where $M=2^{p}$.. We note that:

$$
v_{m+\frac{M}{2}}=-v_{m} .
$$

If we consider $\tilde{\mathcal{V}}_{2^{p}}=\left\{\tilde{v}_{m}=e^{\frac{j 2 \pi m}{M}}, m=1, \ldots, \frac{M}{2}\right\}$, then $\mathcal{V}_{2^{p}}=\tilde{\mathcal{V}}_{2^{p}} \cup\left(-\tilde{\mathcal{V}}_{2^{p}}\right)$. From (19) and given that $\mathcal{V}_{2^{p}}=\mathcal{V}$, the likelihood function can be written as:

$$
\begin{aligned}
\Lambda(u) & =\prod_{k} \sum_{\tilde{v}_{m} \in \tilde{\mathcal{V}}_{2^{p}}}\left[\mathrm{P}\left(a_{k}=\tilde{v}_{m}\right) \exp \left(\frac{\Re\left\{\tilde{v}_{m}^{*} x_{k}(u)\right\}}{\sigma^{2}}\right)\right. \\
& \left.+\mathrm{P}\left(a_{k}=-\tilde{v}_{m}\right) \exp \left(-\frac{\Re\left\{\tilde{v}_{m}^{*} x_{k}(u)\right\}}{\sigma^{2}}\right)\right] .
\end{aligned}
$$


In order to compute $\mathrm{P}\left(a_{k}=\tilde{v}_{m}\right)$, we use a Gray-mapping technique for $M$-PSK modulated signals. According to (16) and (17), the symbol probability is given by:

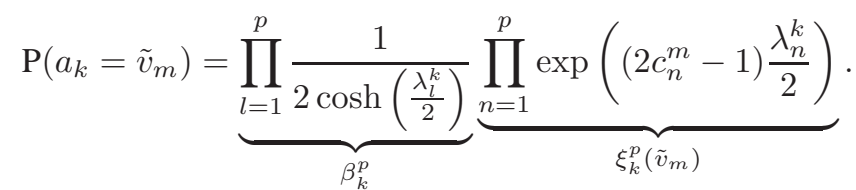

For notation convenience, we briefly describe the recurrence based construction of a $2^{p}$-PSK Gray coded constellation starting from a $2^{p-1}-$ PSK Gray coded constellation.

TABLE. II illustrates the step by step construction of the code, where for a given $p$, each column corresponds to a symbol.

TABLE II: Gray Code For Various Constellation Lengths

\begin{tabular}{|c|c|c|c|c|c|c|c|c|c|c|c|c|c|c|c|c|}
\hline $\bar{p}=1$ & 0 & 1 & & & & & & & & & & & & & & \\
\hline$p=2$ & $\begin{array}{l}0 \\
\underline{0}\end{array}$ & $\begin{array}{l}1 \mid \\
\underline{0}\end{array}$ & $\begin{array}{l}1 \\
\underline{1}\end{array}$ & $\begin{array}{l}0 \\
\underline{1}\end{array}$ & & & & & & & & & & & & \\
\hline$p=3$ & $\begin{array}{l}0 \\
0 \\
\underline{0}\end{array}$ & $\begin{array}{l}1 \\
0 \\
\underline{\mathbf{0}}\end{array}$ & $\begin{array}{l}1 \\
1 \\
\underline{\mathbf{0}}\end{array}$ & $\begin{array}{l}0 \\
1 \\
\underline{0}\end{array}$ & $\begin{array}{l}0 \\
1 \\
\underline{1}\end{array}$ & $\begin{array}{l}1 \\
1 \\
\underline{1}\end{array}$ & $\begin{array}{l}1 \\
0 \\
\underline{1}\end{array}$ & $\begin{array}{l}0 \\
0 \\
\underline{\mathbf{1}}\end{array}$ & & & & & & & & \\
\hline$p=4$ & $\begin{array}{l}0 \\
0 \\
0 \\
0\end{array}$ & $\begin{array}{l}1 \\
0 \\
0 \\
0\end{array}$ & $\begin{array}{l}1 \\
1 \\
0 \\
0\end{array}$ & $\begin{array}{l}0 \\
1 \\
0 \\
\underline{0}\end{array}$ & $\begin{array}{l}0 \\
1 \\
1 \\
0\end{array}$ & $\begin{array}{l}1 \\
1 \\
1 \\
0\end{array}$ & $\begin{array}{l}1 \\
0 \\
1 \\
0\end{array}$ & $\begin{array}{l}0 \\
0 \\
1 \\
0\end{array}$ & $\begin{array}{l}0 \\
0 \\
1 \\
\underline{1}\end{array}$ & $\begin{array}{l}1 \\
0 \\
1 \\
\underline{1}\end{array}$ & $\begin{array}{l}1 \\
1 \\
1 \\
\underline{1}\end{array}$ & $\begin{array}{l}0 \\
1 \\
1 \\
\underline{1}\end{array}$ & $\begin{array}{l}0 \\
1 \\
0 \\
\underline{1}\end{array}$ & $\begin{array}{l}1 \\
1 \\
0 \\
\underline{1}\end{array}$ & $\begin{array}{l}1 \\
0 \\
0 \\
\underline{1}\end{array}$ & $\begin{array}{l}0 \\
0 \\
0 \\
\underline{1}\end{array}$ \\
\hline
\end{tabular}

In this section, we are interested in $p$ values greater than or equal to 3 .

In general, and based on the previous example,

$$
\begin{aligned}
\forall \tilde{v}_{m} \in \tilde{\mathcal{V}}_{2^{p}} \quad & c_{p}^{m}=0, \\
\forall \tilde{v}_{m} \in\left(-\tilde{\mathcal{V}}_{2^{p}}\right) & c_{p}^{m}=1,
\end{aligned}
$$

The $p-1$ MSB of $\tilde{\mathcal{V}}_{2^{p}}$ elements are those of $\tilde{\mathcal{V}}_{2^{p-1}}$ elements and the $p-1$ MSB of $\left(-\tilde{\mathcal{V}}_{2^{p}}\right)$ elements are those of $\tilde{\mathcal{V}}_{2^{p-1}}$ elements but in the reverse order.

The symbol probability (36) becomes:

$$
\mathrm{P}\left(a_{k}=\tilde{v}_{m}\right)=\beta_{k}^{p} \underbrace{\prod_{l=1}^{p-1} \exp \left(\left(2 c_{l}^{m}-1\right) \frac{\lambda_{l}^{k}}{2}\right)}_{\gamma_{k}^{p-1}\left(\tilde{v}_{m}\right)} \exp \left(\left(2 c_{p}^{m}-1\right) \frac{\lambda_{p}^{k}}{2}\right) .
$$

where $\gamma_{k}^{2}\left(\tilde{v}_{m}\right)=1$. As a result, for any $\tilde{v}_{m}$ in $\tilde{\mathcal{V}}_{2^{p}}$ :

$$
\begin{gathered}
\mathrm{P}\left(a_{k}=\tilde{v}_{m}\right)=\beta_{k}^{p} \gamma_{k}^{p-1}\left(\tilde{v}_{m}\right) \exp \left(-\frac{\lambda_{p}^{k}}{2}\right), \\
\mathrm{P}\left(a_{k}=-\tilde{v}_{m}\right)=\beta_{k}^{p} \gamma_{k}^{p-1}\left(-\tilde{v}_{m}\right) \exp \left(\frac{\lambda_{p}^{k}}{2}\right) .
\end{gathered}
$$

Replacing the symbol probability (39) into (35) we obtain:

$$
\begin{aligned}
\Lambda(u)= & \prod_{k} \frac{\beta_{k}^{p}}{\sigma^{2}} \sum_{m=1}^{2^{p-1}}\left[\gamma_{k}^{p-1}\left(\tilde{v}_{m}\right) \exp \left(-\frac{\lambda_{p}^{k}}{2}\right) \times\right. \\
& \exp \left(\frac{\Re\left\{\tilde{v}_{m}^{*} x_{k}(u)\right\}}{\sigma^{2}}\right)+\gamma_{k}^{p-1}\left(-\tilde{v}_{m}\right) \times \\
& \left.\exp \left(\frac{\lambda_{p}^{k}}{2}\right) \exp \left(-\frac{\Re\left\{\tilde{v}_{m}^{*} x_{k}(u)\right\}}{\sigma^{2}}\right)\right],
\end{aligned}
$$

where $\tilde{v}_{m}=\exp \left(j \frac{2 \pi m}{M}\right)$, for $m=1, . ., M / 2=2^{p-1}$.
Here, we are actually dealing with the first $p-1$ most significant bits (MSB) of $\tilde{v}_{m} \Leftrightarrow c_{1}^{m} c_{2}^{m} \ldots c_{p-1}^{m} c_{p}^{m}$. We define $\tilde{v}_{n}$ the $2^{p-1}-\mathrm{PSK}$ symbol associated to the Gray-mapping $\tilde{v}_{n} \Leftrightarrow c_{1}^{m} c_{2}^{m} \ldots c_{p-1}^{m}$, where $n$ is in $\left\{1, \ldots, 2^{p-1}\right\}$. We evoke the same decomposition $\mathcal{V}_{2^{p-1}}=\tilde{\mathcal{V}}_{2^{p-1}} \cup\left(-\tilde{\mathcal{V}}_{2^{p-1}}\right)$ for the $2^{p-1}-\mathrm{PSK}$ constellation mapping. The symbols $\tilde{v}_{n}$ are obtained from the previous Gray-coded $2^{p-1}-$ PSK constellation by applying the same construction procedure. Thus, $\tilde{v}_{n}$ and $-\tilde{v}_{n}$ have the same $(p-2)$ MSBs. This analysis then requires that $p \geq 3$ which justifies the separate treatment of $p=1$ (BPSK) and $p=2$ (QPSK).

Accordingly:

$$
\gamma_{k}^{p-2}\left(\tilde{v}_{n}\right)=\prod_{l=1}^{p-2} \exp \left(\left(2 c_{l}^{m}-1\right) \frac{\lambda_{l}^{k}}{2}\right),
$$

which yields the following expression:

$$
\gamma_{k}^{p-1}\left(\tilde{v}_{m}\right)=\gamma_{k}^{p-2}\left(\tilde{v}_{n}\right) \exp \left(\left(2 c_{p-1}^{m}-1\right) \frac{\lambda_{p-1}^{k}}{2}\right) .
$$

Now, we are going to derive the expression of $c_{p-1}^{m}$ as a function of $m$. We actually know that:

$$
c_{p-1}^{m}=\left\{\begin{array}{l}
0 \text { if } 1 \leq n \leq \frac{2^{p-1}}{2}=2^{p-2} \\
1 \text { if } 2^{p-2}<n \leq 2^{p-1}
\end{array}\right.
$$

Given that:

$$
\begin{aligned}
1 \leq n \leq 2^{p-2} & \Leftrightarrow\left\lfloor\frac{n}{2^{p-2}+1}\right\rfloor=0, \\
2^{p-2}<n \leq 2^{p-1} & \Leftrightarrow\left\lfloor\frac{n}{2^{p-2}+1}\right\rfloor=1,
\end{aligned}
$$

where $\lfloor x\rfloor$ is the integer part of $x$, we can write that:

$$
c_{p-1}^{m}=\left\lfloor\frac{n}{2^{p-2}+1}\right\rfloor .
$$

It is also straightforward to show that:

$$
n=\operatorname{rem}\left(m, \frac{M}{2}\right) \text {, }
$$

where $M=2^{p}$ and $\operatorname{rem}(x, y)$ is the remainder of the Euclidean division of $x$ by $y$. As a result:

$$
c_{p-1}^{m}=\left\lfloor\frac{\operatorname{rem}\left(m, 2^{p-1}\right)}{2^{p-2}+1}\right\rfloor .
$$

Consequently, we obtain that:

$$
\begin{aligned}
\gamma_{k}^{p-1}\left(\tilde{v}_{m}\right)= & \gamma_{k}^{p-2}\left(\tilde{v}_{n}\right) \exp \left(\left(2\left\lfloor\frac{\operatorname{rem}\left(m, 2^{p-1}\right)}{2^{p-2}+1}\right\rfloor-1\right)\right. \\
& \left.\times \frac{\lambda_{p-1}^{k}}{2}\right) \\
= & \alpha_{p-1}^{k}(m),
\end{aligned}
$$

where $\alpha_{p-1}^{k}(m)$ is calculated recursively for any $p \geq 3$ from:

$$
\begin{aligned}
\alpha_{p-1}^{k}(m)= & \alpha_{p-2}^{k}\left(\operatorname{rem}\left(m, 2^{p-1}\right)\right) \times \\
& \exp \left(\left(2\left\lfloor\frac{\operatorname{rem}\left(m, 2^{p-1}\right)}{2^{p-2}+1}\right\rfloor-1\right) \frac{\lambda_{p-1}^{k}}{2}\right) .
\end{aligned}
$$


Due to the construction symmetry of the Gray-mapping, we can note that for any integer $m$ in $\left\{1, \ldots, 2^{p-1}\right\}$ :

$$
\begin{aligned}
\gamma_{k}^{p-1}\left(-\tilde{v}_{m}\right)= & \gamma_{k}^{p-1}\left(\tilde{v}_{2^{p-1}-m+1}\right) \\
= & \alpha_{p-1}^{k}\left(2^{p-1}-m+1\right) \\
= & \alpha_{p-2}^{k}\left(\operatorname{rem}\left(2^{p-1}-m+1,2^{p-1}\right)\right) \times \\
& \exp \left(\left(2\left\lfloor\frac{\operatorname{rem}\left(2^{p-1}-m+1,2^{p-1}\right)}{2^{p-2}+1}\right\rfloor-1\right) \frac{\lambda_{p-1}^{k}}{2}\right) .
\end{aligned}
$$

On one hand we have that:

$$
\alpha_{p-2}^{k}\left(\operatorname{rem}\left(2^{p-1}-m+1,2^{p-1}\right)\right)=\alpha_{p-2}^{k}\left(2^{p-1}-m+1\right) .
$$

On the other hand we have:

$$
\left\lfloor\frac{\operatorname{rem}\left(2^{p-1}-m+1,2^{p-1}\right)}{2^{p-2}+1}\right\rfloor=\left\{\begin{array}{l}
1 \text { if } m \in\left\{1, \ldots, 2^{p-2}\right\}, \\
0 \text { if } m \in\left\{2^{p-2}+1, \ldots, 2^{p-1}\right\} .
\end{array}\right.
$$

Thus:

$$
\gamma_{k}^{p-1}\left(-\tilde{v}_{m}\right)=\left\{\begin{array}{l}
\alpha_{p-2}^{k}\left(2^{p-1}-m+1\right) \exp \left(\frac{\lambda_{p-1}^{k}}{2}\right) \text { if } 1 \leq m \leq 2^{p-2}, \\
\alpha_{p-2}^{k}\left(2^{p-1}-m+1\right) \exp \left(-\frac{\lambda_{p-1}^{k}}{2}\right) \text { if } 2^{p-2}<m \leq 2^{p-1} .
\end{array}\right.
$$

As a result, the likelihood function (42) becomes:

$$
\begin{aligned}
\Lambda(u) & =\prod_{k} \frac{\beta_{k}^{p}}{\sigma^{2}} \sum_{m=1}^{2^{p-2}} \alpha_{p-2}^{k}(m) \exp \left(\frac{\Re\left\{\tilde{v}_{m}^{*} x_{k}(u)\right\}}{\sigma^{2}}-\frac{\lambda_{p-1}^{k}}{2}-\frac{\lambda_{p}^{k}}{2}\right) \\
& +\alpha_{p-2}^{k}\left(2^{p-1}-m+1\right) \exp \left(-\frac{\Re\left\{\tilde{v}_{m}^{*} x_{k}(u)\right\}}{\sigma^{2}}+\frac{\lambda_{p-1}^{k}}{2}+\frac{\lambda_{p}^{k}}{2}\right) \\
& +\sum_{m=2^{p-2}+1}^{2^{p-1}} \alpha_{p-2}^{k}(m) \exp \left(\frac{\Re\left\{\tilde{v}_{m}^{*} x_{k}(u)\right\}}{\sigma^{2}}+\frac{\lambda_{p-1}^{k}}{2}-\frac{\lambda_{p}^{k}}{2}\right) \\
& +\alpha_{p-2}^{k}\left(2^{p-1}-m+1\right) \exp \left(-\frac{\Re\left\{\tilde{v}_{m}^{*} x_{k}(u)\right\}}{\sigma^{2}}-\frac{\lambda_{p-1}^{k}}{2}+\frac{\lambda_{p}^{k}}{2}\right) .
\end{aligned}
$$

Due to the construction symmetry of the Gray mapping, the first $p-2$-bits of any symbol $\tilde{v}_{m}$ are identical to that of $\tilde{v}_{2^{p-1}-m+1}$, for any integer $m$ in $\left\{1,2^{p-1}\right\}$, so that, $\alpha_{p-2}^{k}\left(2^{p-1}-m+1\right)=\alpha_{p-2}^{k}(m)$.

Based on the fact that, $\tilde{v}_{m+2^{p-2}}^{*}=j \tilde{v}_{m}^{*}$, and from (57), the log-likelihood function can be written as:

$$
\begin{aligned}
\Lambda_{L}(u)= & \sum_{k} \log \left(\frac{2 \beta_{k}^{p}}{N_{0}}\right)+\sum_{k} \log \left(\sum_{m=1}^{2^{p-2}} \alpha_{p-2}^{k}(m) \times\right. \\
& \cosh \left(\frac{\Re\left\{\tilde{v}_{m}^{*} x_{k}(u)\right\}}{N_{0}}-\frac{\lambda_{p-1}^{k}}{2}-\frac{\lambda_{p}^{k}}{2}\right) \\
& +\sum_{m=1}^{2^{p-2}} \alpha_{p-2}^{k}\left(2^{p-2}-m+1\right) \times \\
& \left.\cosh \left(-\frac{\Im\left\{\tilde{v}_{m}^{*} x_{k}(u)\right\}}{N_{0}}+\frac{\lambda_{p-1}^{k}}{2}-\frac{\lambda_{p}^{k}}{2}\right)\right),
\end{aligned}
$$

where $N_{0}=\sigma^{2}$ is the power spectral density of the complexvalued AWGN noise.
Let us define:

$$
\begin{aligned}
H_{k}(x) & =\sum_{m=1}^{2^{p-2}} \alpha_{p-2}^{k}(m) \cosh \left(\frac{\Re\left\{\tilde{v}_{m}^{*} x\right\}}{N_{0}}-\frac{\lambda_{p-1}^{k}}{2}-\frac{\lambda_{p}^{k}}{2}\right) \\
& +\alpha_{p-2}^{k}\left(2^{p-2}-m+1\right) \cosh \left(-\frac{\Im\left\{\tilde{v}_{m}^{*} x\right\}}{N_{0}}+\frac{\lambda_{p-1}^{k}}{2}-\frac{\lambda_{p}^{k}}{2}\right)(59) \\
g_{m}(x) & =\alpha_{p-2}^{k}(m) \sinh \left(\frac{\Re\left\{\tilde{v}_{m}^{*} x\right\}}{N_{0}}-\frac{\lambda_{p-1}^{k}}{2}-\frac{\lambda_{p}^{k}}{2}\right) \text { and } \\
h_{m}(x) & =\alpha_{p-2}^{k}\left(2^{p-2}-m+1\right) \sinh \left(-\frac{\Im\left\{\tilde{v}_{m}^{*} x\right\}}{N_{0}}+\frac{\lambda_{p-1}^{k}}{2}-\frac{\lambda_{p}^{k}}{2}\right)(61)
\end{aligned}
$$

From (58)-(61), we find that:

$$
\frac{\partial \Lambda_{L}(u)}{\partial u}=\frac{1}{N_{0}} \sum_{k} \Re\left\{\tilde{a}_{k}^{*}(u) \frac{\partial x_{k}(u)}{\partial u}\right\},
$$

where the real and the imaginary parts $\tilde{a}_{k, 1}$ and $\tilde{a}_{k, 2}$ of the soft symbol are given by:

$$
\tilde{a}_{k, i}(u)=\frac{I_{i}\left(x_{k}(u)\right)}{H_{k}\left(x_{k}(u)\right)}, i \in\{1,2\}
$$

with:

$$
\begin{gathered}
I_{i}(x)=\sum_{m=1}^{2^{p-1}} g_{m}(x) \kappa_{i}+h_{m}(x) \varepsilon_{i}, \\
\kappa_{i}=\left\{\begin{array}{l}
\tilde{v}_{m, 1} \text { if } i=1 \\
-\tilde{v}_{m, 2} \text { if } i=2
\end{array}\right.
\end{gathered}
$$

and:

$$
\varepsilon_{i}=\left\{\begin{array}{l}
\tilde{v}_{m, 2} \text { if } i=1 \\
-\tilde{v}_{m, 1} \text { if } i=2
\end{array}\right.
$$

For $M$-PSK, the timing offset estimate is also updated according to (24) where the real and imaginary parts of $\tilde{a}_{k}$ are given by (63) for $u=\tau_{k-1}$.

\section{CRAMER-RAO BOUnd}

In this section, we derive the Cramer-Rao Bound (CRB) for a code-aided delay estimation in the case of BPSK, QPSK and $M$-PSK $(M \geq 8)$ modulated signals. In this paper derivation of the CRB is conditioned to the LLRs values. It is worth to note that the herein derived $\mathrm{CRB}$ is conditioned to the LLR values. A further averaging over the possible soft output values should be then carried to evaluate the CRB. This is here processed by averaging over Monte Carlo trials. Further work could be carried to develop a semi analytical CRB expression by analytically accounting for the LLRs variation as done in [23] and [33] for the problem of MSE computation.

Suppose that we are able to produce an unbiased estimate $\hat{\tau}$ of the delay $\tau$ from the received signal. The CRB which verifies $E\left[(\hat{\tau}-\tau)^{2}\right] \geq \operatorname{CRB}(\tau)$ for any estimator $\hat{\tau}$ of $\tau$ is defined as [16]:

$$
\operatorname{CRB}(\tau)=I^{-1}(\tau),
$$

where $I(\tau)$ is the Fisher information matrix (FIM) [16] given by:

$$
I(\tau)=E\left[\left(\frac{\partial \Lambda_{L}(\tau)}{\partial \tau}\right)^{2}\right]
$$

In the next paragraph we first evaluate the expression (68) in the case of a BPSK modulated signal. 


\section{A. BPSK modulation}

For convenience, we recall that the log-likelihood function is given by (21). By differentiating $\Lambda_{L}(\tau)$ with respect to $\tau$ and then squaring the result we have that:

$$
\begin{aligned}
\left(\frac{\partial \Lambda_{L}(\tau)}{\partial \tau}\right)^{2} & =\sum_{j} \sum_{i} \frac{2}{N_{0}^{2}} \frac{\partial x_{j, 1}(\tau)}{\partial \tau} \frac{\partial x_{i, 1}(\tau)}{\partial \tau} \\
& \times \tanh \left(\frac{\lambda_{j}}{2}+\frac{2 x_{j, 1}(\tau)}{N_{0}}\right) \tanh \left(\frac{\lambda_{i}}{2}+\frac{2 x_{i, 1}(\tau)}{N_{0}}\right) .
\end{aligned}
$$

Taking the average of equation (69) over the observation noise and the detected symbols, we then obtain:

$$
\begin{aligned}
E\left[\left(\frac{\partial \Lambda_{L}(\tau)}{\partial \tau}\right)^{2}\right] & =\frac{4}{N_{0}^{2}} \sum_{i} E\left[\left(\frac{\partial x_{i, 1}(\tau)}{\partial \tau}\right)^{2}\right. \\
& \left.\times \tanh ^{2}\left(\frac{\lambda_{i}}{2}+\frac{2 x_{i, 1}(\tau)}{N_{0}}\right)\right] \\
& +\frac{4}{N_{0}^{2}} \sum_{j} \sum_{i \neq j} E\left[\frac{\partial x_{j, 1}(\tau)}{\partial \tau} \frac{\partial x_{i, 1}(\tau)}{\partial \tau}\right. \\
& \left.\times \tanh \left(\frac{\lambda_{j}}{2}+\frac{2 x_{j, 1}(\tau)}{N_{0}}\right) \tanh \left(\frac{\lambda_{i}}{2}+\frac{2 x_{i, 1}(\tau)}{N_{0}}\right)\right] .
\end{aligned}
$$

In order to compute the first term of (70), we show in Appendix A, as the demonstration is also useful in the sequel, that $\frac{\partial x_{j, 1}(\tau)}{\partial \tau}$ and $x_{j, 1}(\tau)$ are uncorrelated. Consequently, (70) becomes:

$$
\begin{aligned}
& E\left[\left(\frac{\partial \Lambda_{L}(\tau)}{\partial \tau}\right)^{2}\right] \\
= & \sum_{i} \frac{4}{N_{0}^{2}} E\left[\tanh ^{2}\left(\frac{\lambda_{i}}{2}+\frac{2 x_{i, 1}(\tau)}{N_{0}}\right)\right] E\left[\left(\frac{\partial x_{i, 1}(\tau)}{\partial \tau}\right)^{2}\right] \\
+ & \sum_{j} \sum_{i \neq j} \frac{4}{N_{0}^{2}} E\left[\frac{\partial x_{j, 1}(\tau)}{\partial \tau} \frac{\partial x_{i, 1}(\tau)}{\partial \tau} \times\right. \\
& \left.\tanh \left(\frac{\lambda_{j}}{2}+\frac{2 x_{j, 1}(\tau)}{N_{0}}\right) \tanh \left(\frac{\lambda_{i}}{2}+\frac{2 x_{i, 1}(\tau)}{N_{0}}\right)\right] .
\end{aligned}
$$

The derivation of the various expectations in (71) is given in Appendix B. We finally obtain that:

$$
\begin{aligned}
E\left[\left(\frac{\partial \Lambda_{L}(\tau)}{\partial u}\right)^{2}\right] & =\sum_{i} \frac{4}{N_{0}^{2}}\left[1-\frac{1}{\sqrt{\pi N_{0}}} \int_{-\infty}^{+\infty} G_{i}\left(1, x, N_{0}\right) d x\right] \\
& \times\left[\frac{N_{0}}{2} g^{\prime \prime}(0)+\sum_{n} g^{\prime}(n T)^{2}\right] \\
& -\sum_{i} \sum_{j \neq i} \frac{4}{N_{0}^{2}} g^{\prime}((j-i) T)^{2},
\end{aligned}
$$

where:

$$
G_{i}\left(\nu, x, N_{0}\right)=\frac{\exp \left(-\frac{x^{2}+\nu^{2}}{N_{0}}\right)}{\cosh \left(\frac{2 \nu x}{N_{0}}+\frac{\lambda_{i}}{2}\right)} .
$$

Equation (72) implicitly depends on the SNR and the inverse of (72) gives the CRB in the case of a BPSK modulation scheme.

\section{B. QPSK modulation}

According to (31), squaring the derivative of the $\log$ likelihood function $\Lambda_{L}(\tau)$ with respect to $\tau$, leads to:

$$
\left(\frac{\partial \Lambda_{L}(\tau)}{\partial \tau}\right)^{2}=\sum_{k} \sum_{n} \frac{2}{N_{0}^{2}} \sum_{i=1}^{2} \sum_{l=1}^{2} \tilde{a}_{k, i}(\tau) \tilde{a}_{n, l}(\tau) \frac{\partial x_{k, i}(\tau)}{\partial \tau} \frac{\partial x_{n, l}(\tau)}{\partial \tau} .
$$

From (32) and (33), we know that $\tilde{a}_{k, i}(\tau)$ is function of $x_{k, i}(\tau)$. From Appendix A, we know that $x_{k}(\tau)$ and $\frac{\partial x_{k}(\tau)}{\partial \tau}$ are uncorrelated; considering the real and the imaginary parts of the QPSK symbols as binary modulated signals, $x_{k, i}(\tau)$ and $\frac{\partial x_{k, i}(\tau)}{\partial \tau}$ are similarly uncorrelated. Thus:

$$
\begin{aligned}
& E\left[\left(\frac{\partial \Lambda_{L}(\tau)}{\partial \tau}\right)^{2}\right] \\
= & \frac{2}{N_{0}^{2}} \sum_{k} \sum_{i=1}^{2} E\left[\tilde{a}_{k, i}^{2}(\tau)\right] E\left[\left(\frac{\partial x_{k, i}(\tau)}{\partial \tau}\right)^{2}\right] \\
+ & \sum_{k} \sum_{n, n \neq k} \sum_{i=1}^{2} \sum_{l=1}^{2} \frac{2}{N_{0}^{2}} E\left[\frac{\partial x_{k, i}(\tau)}{\partial \tau} \frac{\partial x_{n, l}(\tau)}{\partial \tau} \tilde{a}_{k, i}(\tau) \tilde{a}_{n, l}(\tau)\right] .
\end{aligned}
$$

Consequently from (32) and (33):

$$
\begin{aligned}
& E\left[\left(\frac{\partial \Lambda_{L}(\tau)}{\partial \tau}\right)^{2}\right] \\
= & \sum_{k} \sum_{i=1}^{2} \frac{2}{N_{0}^{2}} E\left[\tanh ^{2}\left(\frac{\lambda_{i}^{k}}{2}+\frac{2 v_{0, i} x_{k, i}(\tau)}{N_{0}}\right)\right] \times \\
& E\left[\left(\frac{\partial x_{k, i}(\tau)}{\partial \tau}\right)^{2}\right]+\sum_{k} \sum_{n} \frac{2}{N_{0}^{2}} \sum_{i=1}^{2} \sum_{l=1, l \neq i}^{2} \\
& E\left[\frac{\partial x_{k, i}(\tau)}{\partial \tau} \frac{\partial x_{n, l}(\tau)}{\partial \tau} \tanh \left(\frac{\lambda_{i}^{k}}{2}+\frac{2 v_{0, i} x_{k, i}(\tau)}{N_{0}}\right) \times\right. \\
& \left.\tanh \left(\frac{\lambda_{l}^{n}}{2}+\frac{2 v_{0, l} x_{n, l}(\tau)}{N_{0}}\right)\right] .
\end{aligned}
$$

In order to evaluate (75), according to equations (8)-(11) and given that $g$ is a Nyquist filter, we have:

$$
x_{k, 1}(\tau)=a_{k, 1}+\int_{T_{0}} h(t-j T-\tau) \Im\{n(t)\} d t,
$$

and:

$$
x_{k, 2}(\tau)=a_{k, 2}+\int_{T_{0}} h(t-j T-\tau) \Re\{n(t)\} d t .
$$

Thus, $x_{k, 1}(\tau)$ and $x_{k, 2}(\tau)$ are normally distributed and similarly, we obtain the following pdf:

$$
f_{x_{k, 1}(\tau)}(x)=\frac{\exp \left(-\frac{x^{2}+v_{0,1}^{2}}{N_{0}}\right)}{\sqrt{\pi N_{0}} \cosh \left(\frac{\lambda_{1}^{k}}{2}\right)} \cosh \left(\frac{2 x v_{0,1}}{N_{0}}+\frac{\lambda_{1}^{k}}{2}\right),
$$

and:

$$
f_{x_{k, 2}(\tau)}(x)=\frac{\exp \left(-\frac{x^{2}+v_{0,2}^{2}}{N_{0}}\right)}{\sqrt{\pi N_{0}} \cosh \left(\frac{\lambda_{1}^{k}}{2}\right)} \cosh \left(\frac{2 v_{0,2} x}{N_{0}}+\frac{\lambda_{1}^{k}}{2}\right) .
$$

Using the fact $a_{k, 1}$ and $a_{k, 2}$ are considered as binary modulated signals and by analogy to the results found in the case of a BPSK modulation, we obtain the following expressions:

$$
\begin{aligned}
& E\left[\tanh ^{2}\left(\frac{\lambda_{i}^{k}}{2}+\frac{2 v_{0, i} x_{k, i}(\tau)}{N_{0}}\right)\right] \\
= & 1-\frac{1}{\sqrt{\pi N_{0}}} \int_{-\infty}^{+\infty} G_{i}\left(v_{0, i}, x, N_{0}\right) d x,
\end{aligned}
$$


where $G_{i}\left(\nu, x, N_{0}\right)$ is given by (73) and for $i \in\{1,2\}$ :

$$
E\left[\left(\frac{\partial x_{k, i}(\tau)}{\partial \tau}\right)^{2}\right]=\frac{N_{0}}{2} g^{\prime \prime}(0)+E\left[a_{k, i}^{2}\right] \sum_{n} g^{\prime}(n T)^{2} \text {. }
$$

Finally, when $n \neq k$ and $l \neq i$, in Appendix $\mathrm{C}$, we show that:

$$
\begin{aligned}
& E\left[\frac{\partial x_{k, i}(\tau)}{\partial \tau} \frac{\partial x_{n, l}(\tau)}{\partial \tau} \tanh \left(\frac{\lambda_{i}^{k}}{2}+\frac{2 v_{0, i} x_{k, i}(\tau)}{N_{0}}\right)\right. \\
& \left.\times \tanh \left(\frac{\lambda_{l}^{n}}{2}+\frac{2 v_{0, l} x_{n, l}(\tau)}{N_{0}}\right)\right]=-2 v_{0, i}{ }^{\prime}((k-n) T)^{2} .
\end{aligned}
$$

Feeding (80), (81) and (82) into (75):

$$
\begin{aligned}
E\left[\left(\frac{\partial \Lambda_{L}(\tau)}{\partial u}\right)^{2}\right] & =\sum_{k} \sum_{i=1}^{2} \frac{2}{N_{0}^{2}}\left[1-\frac{1}{\sqrt{\pi N_{0}}} \int_{-\infty}^{+\infty} G_{k}\left(v_{0, i}, x, N_{0}\right) d x\right] \\
& \times\left[\frac{N_{0}}{2} g^{\prime \prime}(0)+E\left[a_{k, i}^{2}\right] \sum_{n} g^{\prime}(n T)^{2}\right] \\
& -\sum_{k} \sum_{n}\left(\frac{4}{N_{0}}\right)^{2} g^{\prime}((k-n) T)^{2} .
\end{aligned}
$$

Taking the inverse of (83) leads to the CRB of the QPSK modulated signal code-aided timing delay estimation.

\section{M-PSK modulation}

From (62) and (74) we have that:

$$
\begin{aligned}
& E\left[\left(\frac{\partial \Lambda_{L}(\tau)}{\partial \tau}\right)^{2}\right] \\
= & \frac{1}{N_{0}^{2}}\left(\sum_{k} \sum_{i=1}^{2} E\left[\tilde{a}_{k, i}^{2}(\tau)\right] E\left[\left(\frac{\partial x_{k, i}(\tau)}{\partial \tau}\right)^{2}\right]\right. \\
+ & \left.\sum_{k} \sum_{n, n \neq k} \sum_{i=1}^{2} \sum_{l=1}^{2} E\left[\frac{\partial x_{k, i}(\tau)}{\partial \tau} \frac{\partial x_{n, l}(\tau)}{\partial \tau} \tilde{a}_{k, i}(\tau) \tilde{a}_{n, l}(\tau)\right]\right),
\end{aligned}
$$

where the soft estimated $M$-PSK symbol is given by (63). The first term of (84) can be evaluated from (63):

$$
E\left[\tilde{a}_{k, i}^{2}(\tau)\right]=\int \frac{\left(I_{i}(x)\right)^{2}}{H_{k}^{2}(x)} f_{x_{k}(\tau)}(x) d x .
$$

Similarly to Appendix B, the probability density function of $x_{k}(\tau)$ is:

$$
\begin{aligned}
& f_{x_{k}(\tau)}(x) \\
= & \frac{1}{2 \pi N_{0}}\left[\sum_{m=1}^{2^{p-1}} \mathrm{P}\left(a_{k}=\tilde{v}_{m}\right) \exp \left(-\frac{\left|x-\tilde{v}_{m}\right|^{2}}{2 N_{0}}\right)\right. \\
+ & \left.\mathrm{P}\left(a_{k}=-\tilde{v}_{m}\right) \exp \left(-\frac{\left|x+\tilde{v}_{m}\right|^{2}}{2 N_{0}}\right)\right] \\
= & \frac{\exp \left(-\frac{|x|^{2}+1}{2 N_{0}}\right)}{2 \pi N_{0}}\left[\sum_{m=1}^{2^{p-1}} \mathrm{P}\left(a_{k}=\tilde{v}_{m}\right) \exp \left(\frac{\Re\left\{\tilde{v}_{m}^{*} x\right\}}{N_{0}}\right)\right. \\
+ & \left.\mathrm{P}\left(a_{k}=-\tilde{v}_{m}\right) \exp \left(\frac{\Re\left\{\tilde{v}_{m}^{*} x\right\}}{N_{0}}\right)\right] \\
= & \frac{\beta_{k}^{p}}{\pi N_{0}} \exp \left(-\frac{|x|^{2}+1}{2 N_{0}}\right) H_{k}(x) .
\end{aligned}
$$

According to equations (8)-(11) and considering that the transmitted symbols are mutually independent, we have:

$$
E\left[\left(\frac{\partial x_{k, i}(\tau)}{\partial \tau}\right)^{2}\right]=\frac{N_{0}}{2} g^{\prime \prime}(0)+\sum_{n} E\left[a_{k-n, i}^{2}\right] g^{\prime}(n T)^{2}(86)
$$

and:

$$
\begin{aligned}
& E\left[\frac{\partial x_{k, i}(\tau)}{\partial \tau} \frac{\partial x_{n, l}(\tau)}{\partial \tau} \tilde{a}_{k, i}(\tau) \tilde{a}_{n, l}(\tau)\right] \\
= & -\left(\int \frac{I_{i}(x)}{H_{k}(x)} f_{x_{k}(\tau)}(x) d x\right)^{2} g^{\prime}((k-n) T)^{2} \\
= & -\left(\frac{\beta_{k}^{p} g^{\prime}((k-n) T)}{\pi \sigma^{2}} \int I_{i}(x) \exp \left(-\frac{|x|^{2}+1}{2 \sigma^{2}}\right) d x\right)^{2} .
\end{aligned}
$$

We then obtain the expression of the CRB by introducing (85), (86) and (87) in the inverse of (84).

\section{Simulation Results}

In this section, we display the simulation results of the proposed CA time delay estimation algorithms in terms of MSE and compare them first to the DA (ML TED) and the NDA (ML TED) modes, then to the above derived CramerRao bounds.

We consider the case of BPSK, QPSK and 8-PSK signals with an up-sampling factor equal to 8, passed through a raised cosine filter, with a roll-off factor $\alpha$. The turbo-code is composed of two identical Recursive Systematic Coders (RSC) concatenated in parallel with systematic rate $r=1 / 2$ and generator polynomials $(1,0,1,1)$ and $(1,1,0,1)$. A large interleaver is placed between the two RSCs. Results are averaged on blocks of 500 symbols each, over 1000 Monte Carlo iterations. The values of $x_{k}\left(\tau_{k-1}\right)$ are obtained via a classical quadratic interpolation [34]. The LLR values are initialized by the soft demapper outputs. The turbo-decoder's outputs are reinjected only once to the synchronizer's input. $\hat{\tau}_{k}$ is initialized to 0 and its estimated value is depicted at the end of the block (after 500 samples) when the steady state is achieved. Simulation results are evaluated for the time delay $\tau=0.2 T$. The step-size $\mu$ is chosen so as to minimize the MSE; in theory, an adaptive step-size [35], [36] can be obtained to benefit both from fast convergence and low MSE steady-state; however for simplicity reasons, in practice typical fixed values for $\mu$ span between 0.15 and 0.45 according to the possible scenarios.

The involved integrand functions in (72), (83), (85) and (87) decrease rapidly as $x$ increases. Therefore, the integrals over ] $-\infty,+\infty$ [ can be accurately approximated by a finite integral over an interval $[-A,+A]$ and the Riemann integration method can be adequately used. For the following simulation results we have chosen $A=100$ and an integration step which is equal to $1 / 2000$. The evaluation of the CRB is thus possible as all implied expressions are derived.

Figures 1 and 2 show the evolution of the MSE at each time index, for various CA timing detectors and different modulations. The first statement is that all the detectors converge rapidly to the steady state (between 15 and 25 samples). We also see that the MLD offers the best performance; however it is the most complex detector to implement. We further note that the MSE at the steady state depends on the roll-off factor value for the same estimator. This is due to the fact that the effect of the transmission and the matched filters differs from a detector updating term to another (see TABLE I). It is also shown that, the MSE is deteriorated by increasing the modulation order. 


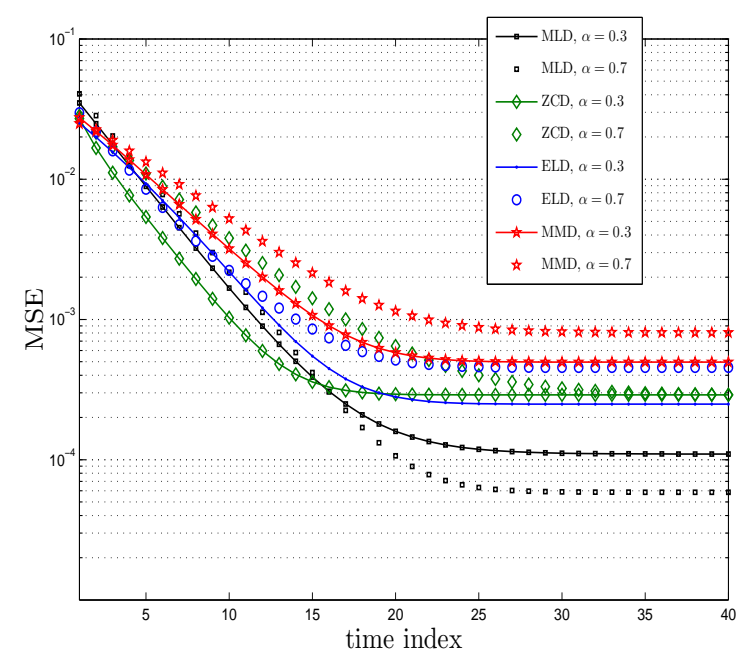

Fig. 1: MSE vs time index for various CA detectors and rolloff factors for BPSK signals $(\mathrm{SNR}=10 \mathrm{~dB})$

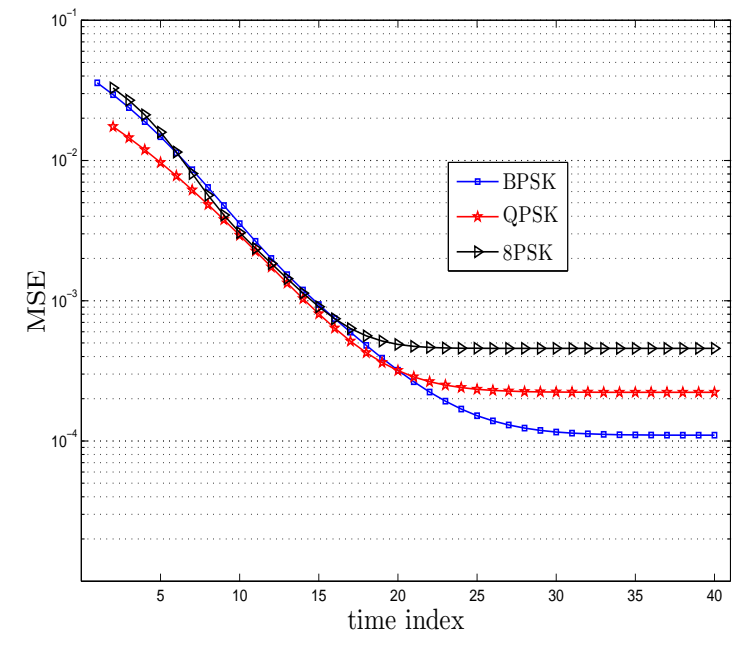

Fig. 2: MSE vs time index for various modulations (ML detector, $\mathrm{SNR}=10 \mathrm{~dB}, \alpha=0.3)$

Fig. 3 depicts the evaluated theoretical expression of the CRB presented in section IV and their empirical CRB counterparts obtained by an averaging over constellation symbols through a Monte Carlo simulation for BPSK, QPSK and 8-PSK signals. We can confirm that the analytical values perfectly match the simulated values of the CRB thus assessing the validity of the derived CRB.

In Fig. 4, 5 and 6, we display results of the CRB and MSE on $(\tau / T)$ as function of the SNR for BPSK, QPSK and 8-PSK modulated signals respectively using a ML based detector. The MSE value is evaluated at the steady state, at the end of the observation block containing 500 symbols. We note that before saturation at high SNRs, the MSE is inversely proportional to the SNR. For each figure, one can compare the estimation performance of the DA, CA and NDA mode. Compared to the NDA mode, the MSE is decreased by exploiting the soft-information from the turbo-decoder. The

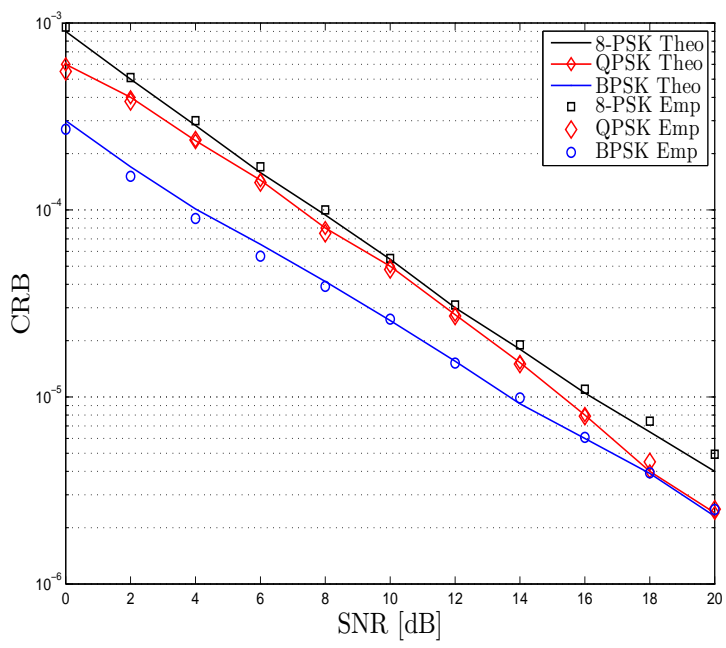

Fig. 3: Comparison between the empirical CRB and the analytical expression for different modulations

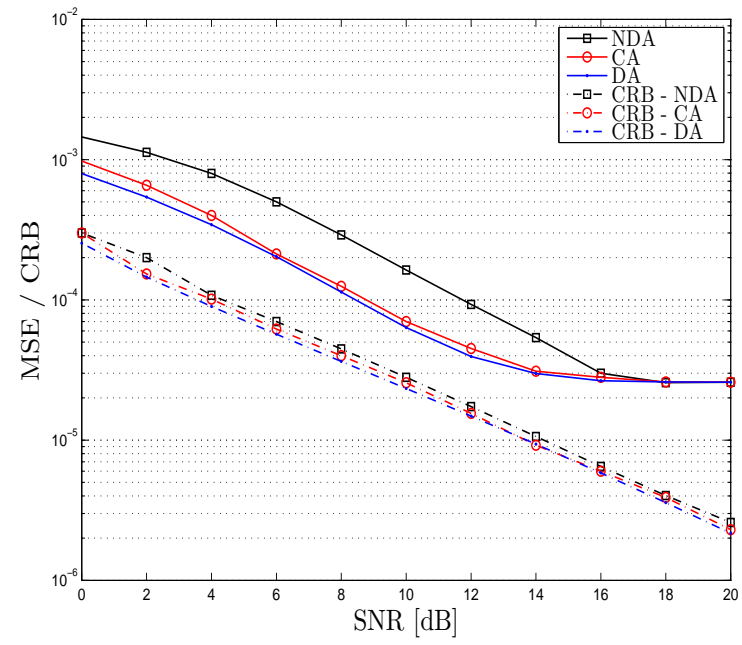

Fig. 4: MSE and CRB vs SNR for a BPSK modulated signal, $\alpha=0.3$

DA mode still achieves the best performance, however, it leads to a higher loss of spectral efficiency. Besides, over a wide range of SNRs, the CA mode performance is almost equivalent to that of the DA mode with no need for pilot symbols. At high SNR the curves related to the 3 synchronization modes merge. The saturation of the MSE at the right side of Fig. 4, 5 and 6 is due to the self noise of the updating error (13). Similar results are obtained for other roll-off factors and other detectors. Some numerical results are summarized in TABLE III and IV.

TABLE III: MSE values for $\mathrm{SNR}=10 \mathrm{~dB}$

\begin{tabular}{|c|c|c|c|}
\hline Modulation & DA & Soft & NDA \\
\hline BPSK & $0.63 \times 10^{-4}$ & $0.7 \times 10^{-4}$ & $1.7 \times 10^{-4}$ \\
\hline QPSK & $1.2 \times 10^{-4}$ & $1.6 \times 10^{-4}$ & $2.5 \times 10^{-4}$ \\
\hline 8-PSK & $1.8 \times 10^{-4}$ & $2.8 \times 10^{-4}$ & $8 \times 10^{-4}$ \\
\hline
\end{tabular}




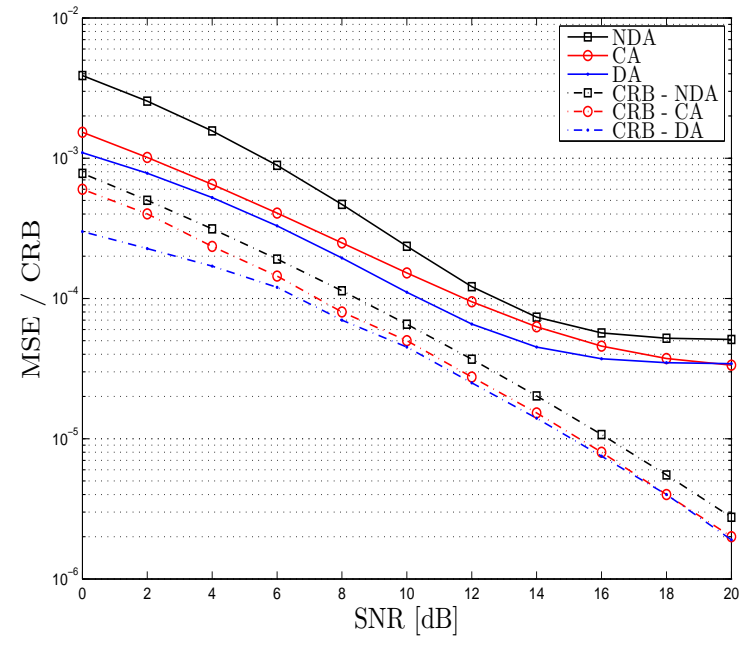

Fig. 5: MSE and CRB vs SNR for a QPSK modulated signal, $\alpha=0.3$

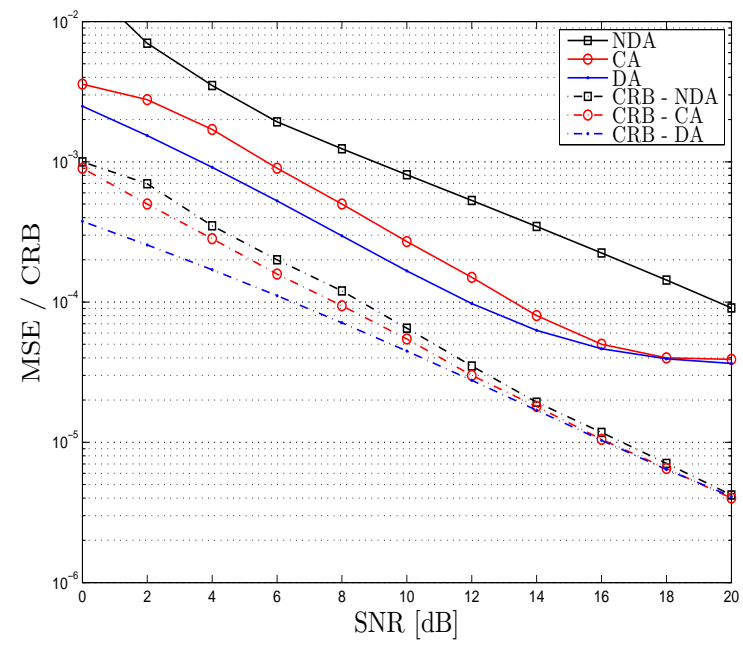

Fig. 6: MSE and CRB vs SNR for a 8-PSK modulated signal, $\alpha=0.3$

\section{CONCLUSION}

In this paper, we studied a new code-aided estimation algorithm for time delay recovery of PSK, QPSK and $M$ PSK modulated signals. The proposed CA algorithm performs better than the NDA algorithm and is near the DA performance over a large interval of SNR values with no need for reference signals. We also established the analytical expression of the CRB for code aided time delay estimation for PSK, QPSK and $M$-PSK signals. The derived expressions validity is corroborated by the empirical CRB counterparts obtained

TABLE IV: SNR values for MSE $=10^{-4}$

\begin{tabular}{|c|c|c|c|}
\hline Modulation & DA & Soft & NDA \\
\hline BPSK & $8.5 \mathrm{~dB}$ & $9 \mathrm{~dB}$ & $12 \mathrm{~dB}$ \\
\hline QPSK & $11 \mathrm{~dB}$ & $12 \mathrm{~dB}$ & $13 \mathrm{~dB}$ \\
\hline 8-PSK & $12 \mathrm{~dB}$ & $14 \mathrm{~dB}$ & $19.5 \mathrm{~dB}$ \\
\hline
\end{tabular}

by simulations. The derived CA CRB provides an absolute benchmark to appreciate the proposed CA estimator relevance.

\section{APPENDIX A \\ PROOF THAT $\frac{\partial x_{j, 1}}{\partial \tau}$ AND $x_{j, 1}$ ARE NOT CORRELATED}

From equation (8), we have that:

$$
\begin{aligned}
x_{j, 1}(\tau) & =\sum_{i} a_{j-i} g(i T)+\int_{T_{0}} h^{*}(t-j T-\tau) \Re\{n(t)\} d t \\
& =a_{j}+\int_{T_{0}} h^{*}(t-j T-\tau) \Re\{n(t)\} d t
\end{aligned}
$$

as $g(t)$ is a Nyquist filter and $g(0)=1$. Also:

$$
\frac{\partial x_{j, 1}(\tau)}{\partial \tau}=\sum_{i} a_{j-i} g^{\prime}(i T)-\int_{T_{0}} h^{\prime}(t-j T-\tau) \Re\{n(t)\} d t .
$$

Let us adopt the following notations:

$$
\begin{aligned}
& \alpha_{j}=\int_{T_{0}} h(t-j T-\tau) \Re\{n(t)\} d t \\
& \dot{\alpha}_{j}=\int_{T_{0}} h^{\prime}(t-j T-\tau) \Re\{n(t)\} d t .
\end{aligned}
$$

We have that:

$$
E\left[x_{j, 1}(\tau) \frac{\partial x_{j, 1}(\tau)}{\partial \tau}\right]=E\left[a_{j} \sum_{i} a_{j-i} g(i T)\right]-E\left[\alpha_{j} \dot{\alpha}_{j}\right]
$$

On one hand:

$$
\begin{aligned}
E\left[\alpha_{j} \dot{\alpha}_{j}\right] & =E\left[\int_{T_{0}} h(t-j T-\tau) \Re\{n(t)\} d t\right. \\
& \left.\times \int_{T_{0}} h^{\prime}(t-j T-\tau) \Re\{n(t)\} d t\right] \\
& =\frac{N_{0}}{2} g^{\prime}(0) \\
& =0 .
\end{aligned}
$$

On the other hand, since $g^{\prime}(0)=0(g(x)$ is maximum in 0) then $\sum_{i} a_{j-i} g^{\prime}(i T)$ and $a_{j}$ are uncorrelated so that $E\left[a_{j} \sum_{i} a_{j-i} g(i T)\right]=0$. Then, $\frac{\partial x_{j, 1}(\tau)}{\partial \tau}$ and $x_{j, 1}(\tau)$ are uncorrelated.

\section{APPENDIX B}

EVALUATION OF THE VARIOUS EXPECTATIONS IN (71)

Derivation of $E\left(\tanh ^{2}\left(\frac{\lambda_{i}}{2}+\frac{2 x_{i, 1}(\tau)}{N_{0}}\right)\right)$ :

From (88), the probability density function (pdf) of $x_{i, 1}(\tau)$ is given by:

$$
\begin{aligned}
f_{x_{i, 1}(\tau)}(x)= & \frac{1}{\sqrt{\pi N_{0}}}\left[\frac{\exp \left(\frac{\lambda_{i}}{2}\right)}{2 \cosh \left(\frac{\lambda_{i}}{2}\right)} \exp \left(-\frac{(x-1)^{2}}{N_{0}}\right)\right. \\
& \left.+\frac{\exp \left(-\frac{\lambda_{i}}{2}\right)}{2 \cosh \left(\frac{\lambda_{i}}{2}\right)} \exp \left(-\frac{(x+1)^{2}}{N_{0}}\right)\right] \\
= & \frac{1}{\sqrt{\pi N_{0}}} \frac{\exp \left(-\frac{x^{2}+1}{N_{0}}\right)}{\cosh \left(\frac{\lambda_{i}}{2}\right)} \cosh \left(\frac{2 x}{N_{0}}+\frac{\lambda_{i}}{2}\right) .
\end{aligned}
$$


We then obtain:

$$
\begin{aligned}
E\left[\tanh ^{2}\left(\frac{\lambda_{i}}{2}+\frac{2 x_{i, 1}(\tau)}{N_{0}}\right)\right] & =\int_{-\infty}^{+\infty} \frac{\tanh ^{2}\left(\frac{\lambda_{i}}{2}+\frac{2 x}{N_{0}}\right)}{\cosh \left(\frac{\lambda_{i}}{2}\right) \sqrt{\pi N_{0}}} \\
& \times \exp \left(-\frac{x^{2}+1}{N_{0}}\right) \cosh \left(\frac{2 x}{N_{0}}+\frac{\lambda_{i}}{2}\right) d x
\end{aligned}
$$

then we average the resulting expression with respect to these two random variables.

We have for any index $i$ and $j$ with $i \neq j$ :

$$
x_{i, 1}(\tau)=a_{i}+\alpha_{i}
$$

Given that $\tanh ^{2}(x)=1-\frac{1}{\cosh ^{2}(x)}$, then:

$$
\begin{aligned}
& E\left[\tanh ^{2}\left(\frac{\lambda_{i}}{2}+\frac{2 x_{i, 1}(\tau)}{N_{0}}\right)\right] \\
= & \frac{1}{\cosh \left(\frac{\lambda_{i}}{2}\right) \sqrt{\pi N_{0}}}\left(\int_{-\infty}^{+\infty} \exp \left(-\frac{x^{2}+1}{N_{0}}\right) \times\right. \\
& \left.\cosh \left(\frac{2 x}{N_{0}}+\frac{\lambda_{i}}{2}\right) d x-\int_{-\infty}^{+\infty} \frac{\exp \left(-\frac{x^{2}+1}{N_{0}}\right)}{\cosh \left(\frac{2 x}{N_{0}}+\frac{\lambda_{j}}{2}\right)} d x\right) .
\end{aligned}
$$

Using the following relation $\cosh (x+y)=\cosh (x) \cosh (y)+$ $\sinh (x) \sinh (y)$, the first integral in (94) is such that:

$$
\begin{aligned}
& \int_{-\infty}^{+\infty} \exp \left(-\frac{x^{2}+1}{N_{0}}\right) \cosh \left(\frac{2 x}{N_{0}}+\frac{\lambda_{i}}{2}\right) d x \\
= & \exp \left(-\frac{1}{N_{0}}\right)\left[\int_{-\infty}^{+\infty} \exp \left(-\frac{x^{2}}{N_{0}}\right) \cosh \left(\frac{2 x}{N_{0}}\right)\right. \\
& \cosh \left(\frac{\lambda_{i}}{2}\right) d x+\sinh \left(\frac{\lambda_{i}}{2}\right) \int_{-\infty}^{+\infty} \exp \left(-\frac{x^{2}}{N_{0}}\right) \\
& \left.\times \sinh \left(\frac{2 x}{N_{0}}\right) d x\right] .
\end{aligned}
$$

The second term of the last summation is the integral of an odd function and it is thus equal to 0. According to [37], we have:

$$
\int_{-\infty}^{+\infty} \exp \left(-\beta x^{2}\right) \cosh (a x) d x=\sqrt{\frac{\pi}{\beta}} \exp \left(\frac{a^{2}}{4 \beta}\right),
$$

so that the first term of (95) is:

$$
\exp \left(-\frac{1}{N_{0}}\right) \int_{-\infty}^{+\infty} \exp \left(-\frac{x^{2}}{N_{0}}\right) \cosh \left(\frac{2 x}{N_{0}}\right) d x=\sqrt{\pi N_{0}} \text {. }
$$

Thus equation (94) becomes:

$$
E\left[\tanh ^{2}\left(\frac{\lambda_{i}}{2}+\frac{x_{i, 1}(\tau)}{N_{0}}\right)\right]=1-\frac{1}{\sqrt{\pi N_{0}}} \int_{-\infty}^{+\infty} G_{i}\left(1, x, N_{0}\right) d x,
$$

where $G_{i}\left(1, x, N_{0}\right)$ is given by (73) for $\nu=1$.

Derivation of $E\left[\left(\frac{\partial x_{i, 1}(\tau)}{\partial \tau}\right)^{2}\right]$ :

Based on the expression of $\frac{\partial x_{i, 1}(\tau)}{\partial \tau}$ in (89) and by averaging over the observations and the symbols we directly obtain:

$$
E\left[\left(\frac{\partial x_{i, 1}(\tau)}{\partial \tau}\right)^{2}\right]=\sum_{n} g^{\prime}(n T)^{2}+\frac{N_{0}}{2} g^{\prime \prime}(0) .
$$

\section{Derivation of}

$$
\begin{aligned}
& E\left[\frac{\partial x_{j, 1}(\tau)}{\partial \tau} \frac{\partial x_{i, 1}(\tau)}{\partial \tau} \tanh \left(\frac{\lambda_{j}}{2}+\frac{2 x_{j, 1}(\tau)}{N_{0}}\right)\right. \\
& \left.\tanh \left(\frac{\lambda_{i}}{2}+\frac{2 x_{i, 1}(\tau)}{N_{0}}\right)\right]:
\end{aligned}
$$

Given that $\frac{\partial x_{j, 1}(\tau)}{\partial \tau}$ and $\frac{\partial x_{i, 1}(\tau)}{\partial \tau}$ are statistically dependent on $x_{i, 1}(\tau)$ and $x_{j, 1}(\tau)$, in order to derive the desired expectation, we first average by conditioning on $x_{i, 1}(\tau)$ and $x_{j, 1}(\tau)$, where $\alpha_{i}$ is given by (90) and:

$$
\begin{aligned}
\frac{\partial x_{j, 1}(\tau)}{\partial \tau} & =\sum_{n} a_{n} g^{\prime}((j-n) T)+\dot{\alpha}_{j} \\
& =a_{i} g^{\prime}((j-i) T)+\sum_{n, n \neq i, j} a_{n} g^{\prime}((j-n) T)+\dot{\alpha}_{j} \\
& =\left[x_{i, 1}(\tau)-\alpha_{i}\right] g^{\prime}((j-i) T)+\sum_{n, n \neq i, j} a_{n} g^{\prime}((j-n) T)+\dot{\alpha}_{j}
\end{aligned}
$$

We then find that:

$$
\begin{aligned}
& E\left[\frac{\partial x_{i, 1}(\tau)}{\partial \tau} \frac{\partial x_{j, 1}(\tau)}{\partial \tau} \mid x_{i, 1}(\tau), x_{j, 1}(\tau)\right] \\
= & x_{i, 1}(\tau) g^{\prime}((j-i) T) x_{j, 1}(\tau) g^{\prime}((i-j) T) \\
= & -x_{i, 1}(\tau) x_{j, 1}(\tau) g^{\prime}((i-j) T)^{2},
\end{aligned}
$$

so that:

$$
\begin{aligned}
& E\left[\frac{\partial x_{i, 1}(\tau)}{\partial \tau} \frac{\partial x_{j, 1}(\tau)}{\partial \tau} \tanh \left(\frac{\lambda_{j}}{2}+\frac{2 x_{j, 1}(\tau)}{N_{0}}\right)\right. \\
& \left.\times \tanh \left(\frac{\lambda_{i}}{2}+\frac{2 x_{i, 1}(\tau)}{N_{0}}\right) \mid x_{i, 1}(\tau), x_{j, 1}(\tau)\right] \\
= & -\tanh \left(\frac{\lambda_{j}}{2}+\frac{2 x_{j, 1}(\tau)}{N_{0}}\right) \tanh \left(\frac{\lambda_{i}}{2}+\frac{2 x_{i, 1}(\tau)}{N_{0}}\right) \\
& \times x_{i, 1}(\tau) x_{j, 1}(\tau) g^{\prime}((i-j) T)^{2} .
\end{aligned}
$$

Finally, we obtain:

$$
\begin{aligned}
& E\left[\frac{\partial x_{i, 1}(\tau)}{\partial \tau} \frac{\partial x_{j, 1}(\tau)}{\partial \tau} \tanh \left(\frac{\lambda_{i}}{2}+\frac{2 x_{i, 1}(\tau)}{N_{0}}\right)\right. \\
& \left.\times \tanh \left(\frac{\lambda_{j}}{2}+\frac{2 x_{j, 1}(\tau)}{N_{0}}\right)\right] \\
= & -E\left[x_{j, 1}(\tau) \tanh \left(\frac{\lambda_{j}}{2}+\frac{2 x_{j, 1}(\tau)}{N_{0}}\right)\right]^{2} g^{\prime}((i-j) T)^{2},
\end{aligned}
$$

where the first term is such that:

$$
\begin{aligned}
& E\left[x_{j, 1}(\tau) \tanh \left(\frac{\lambda_{j}}{2}+\frac{2 x_{j, 1}(\tau)}{N_{0}}\right)\right] \\
= & \int_{-\infty}^{+\infty} x \tanh \left(\frac{\lambda_{j}}{2}+\frac{2 x}{N_{0}}\right) f_{x_{j, 1}(\tau)}(x) d x \\
= & \frac{\exp \left(-\frac{1}{N_{0}}\right)}{\sqrt{\pi N_{0}} \cosh \left(\frac{\lambda_{j}}{2}\right)} \int_{-\infty}^{+\infty} x \sinh \left(\frac{\lambda_{j}}{2}+\frac{2 x}{N_{0}}\right) \exp \left(-\frac{x^{2}}{N_{0}}\right) d x \\
= & 1 .
\end{aligned}
$$

So:

$$
\begin{array}{r}
E\left[\frac{\partial x_{i, 1}(\tau)}{\partial \tau} \frac{\partial x_{j, 1}(\tau)}{\partial \tau} \tanh \left(\frac{\lambda_{i}}{2}+\frac{2 x_{i, 1}(\tau)}{N_{0}}\right)\right. \\
\left.\times \quad \tanh \left(\frac{\lambda_{j}}{2}+\frac{2 x_{j, 1}(\tau)}{N_{0}}\right)\right]=-g^{\prime}((i-j) T)^{2}
\end{array}
$$




\section{APPENDIX C}

\section{DERIVATION OF EQUATION (82)}

We have:

$$
\begin{aligned}
& E\left[\frac{\partial x_{k, i}(\tau)}{\partial \tau} \frac{\partial x_{n, l}(\tau)}{\partial \tau} \tanh \left(\frac{\lambda_{i}^{k}}{2}+\frac{2 v_{0, i} x_{k, i}(\tau)}{N_{0}}\right)\right. \\
& \left.\times \tanh \left(\frac{\lambda_{l}^{n}}{2}+\frac{2 v_{0, l} x_{n, l}(\tau)}{N_{0}}\right)\right] \\
= & -E\left[x_{k, i}(\tau) \tanh \left(\frac{\lambda_{i}^{k}}{2}+\frac{2 v_{0, i} x_{k, i}(\tau)}{N_{0}}\right)\right]^{2} g^{\prime}((k-n) T)^{2} \\
= & -\left[\frac{\exp \left(-\frac{v_{0, i}^{2}}{N_{0}}\right)}{\sqrt{\pi N_{0}} \cosh \left(\frac{\lambda_{i}^{k}}{2}\right)} \int_{-\infty}^{+\infty} x \sinh \left(\frac{\lambda_{i}^{k}}{2}+\frac{2 v_{0, i} x}{N_{0}}\right)\right. \\
& \left.\exp \left(-\frac{x^{2}}{N_{0}}\right) d x g^{\prime}((k-n) T)\right]^{2} .
\end{aligned}
$$

Given that:

$$
\begin{aligned}
& \int_{-\infty}^{+\infty} x \sinh \left(\frac{\lambda_{i}^{k}}{2}+\frac{2 v_{0, i} x}{N_{0}}\right) \exp \left(-\frac{x^{2}}{N_{0}}\right) d x \\
= & \sqrt{\pi N_{0}} \cosh \left(\frac{\lambda_{i}^{k}}{2}\right) \exp \left(\frac{v_{0, i}^{2}}{N_{0}}\right) 2 v_{0, i},
\end{aligned}
$$

and using the fact that $v_{0, i}=1 / \sqrt{2}$, we find:

$$
\begin{aligned}
& E\left[\frac{\partial x_{k, i}(\tau)}{\partial \tau} \frac{\partial x_{n, l}(\tau)}{\partial \tau} \tanh \left(\frac{\lambda_{i}^{k}}{2}+\frac{2 v_{0, i} x_{k, i}(\tau)}{N_{0}}\right)\right. \\
& \left.\times \tanh \left(\frac{\lambda_{l}^{n}}{2}+\frac{2 v_{0, l} x_{n, l}(\tau)}{N_{0}}\right)\right]=-2 g^{\prime}((k-n) T)^{2} .
\end{aligned}
$$

\section{REFERENCES}

[1] K. Mueller and M. Muller, "Timing recovery in digital synchronous data receivers," IEEE Trans. on Communications, vol. 24, no. 5, pp. 516-531, 1976.

[2] F. M. Gardner, "A BPSK/QPSK timing-error detector for sampled receivers," IEEE Trans. on Communications, vol. 34, no. 5, pp. 423 429, 1986.

[3] R. Gallager, "Low-density parity-check codes," IEEE Trans. on Information Theory, vol. 8, no. 1, pp. 21-28, Jan. 1962.

[4] C. Berrou and A. Glavieux, "Near optimum error correcting coding and decoding: turbo-codes," IEEE Trans. on Communications, vol. 44, no. 10, pp. 1261-1271, Oct. 1996.

[5] C. Vanstraceele, B. Geller, J. P. Barbot, and J. M. Brossier, "A low complexity block turbo decoder architecture," IEEE Trans. on Communications, vol. 56, no. 12, pp. 1985-1989, Dec. 2008.

[6] B. Geller, I. Diatta, J. P. Barbot, C. Vanstraceele, and F. Rambeau, "Block turbo codes: From architecture to application," in Proc. of IEEE International Symposium on Information Theory, ISIT 2006, Seattle, Jul. 2006, pp. 1813-1816.

[7] S. Lin and D. J. Costello, Error Control Coding, Second Edition. Upper Saddle River, NJ, USA: Prentice-Hall, Inc., 2004.

[8] C. Berrou, J. Hagenauer, M. Luise, L. Vandendorpe, and C. Schegel, "Turbo-Information processing: algorithms, implementations \& applications," Proc. of the IEEE, vol. 95, no. 6, pp. 1146 - 1149, Jun. 2007.

[9] V. Lottici and M. Luise, "Carrier phase recovery for turbo-coded linear modulations," IEEE International Conference on Communications, ICC, vol. 3, pp. 1541-1545, 2002.

[10] N. Noels, C. Herzet, A. Dejonghe, V. Lottici, H. Steendam, M. Moeneclaey, M. Luise, and L. Vandendorpe, "Turbo synchronization: an EM algorithm interpretation," IEEE International Conference on Communications, ICC, vol. 4, pp. 2933-2937, 2003.

[11] L. Zhang and A. Burr, "APPA symbol timing recovery scheme for turbo codes," PIMRC, vol. 1, pp. 44-48 vol.1, Sep. 2002.

[12] A. Nayak, J. Barry, and S. McLaughlin, "Joint timing recovery and turbo equalization for coded partial response channels," IEEE Trans. on Magnetics, vol. 38, no. 5, pp. 2295-2297, Sep. 2002.
[13] R. Barry, A. Kavcic, S. McLaughlin, A. Nayak, and W. Zeng, "Iterative timing recovery," IEEE Signal Processing Magazine, vol. 21, no. 1, pp. 89-102, Jan. 2004.

[14] I. Nasr, L. Atallah, S. Cherif, B. Geller, and J. Yang, "A soft maximum likelihood technique for time delay recovery," in 2014 International Conference on Communications and Networking (ComNet), Mar. 2014, pp. $1-5$.

[15] I. Nasr, L. Atallah, B. Geller, and S. Cherif, "CRB derivation and new code-aided timing recovery technique for QAM modulated signals," in 2015 IEEE International Conference on Communications (ICC), Jun. 2015, pp. 4901-4906.

[16] S. M. Kay, Fundamentals of Statistical Signal Processing: Estimation Theory. Upper Saddle River, NJ, USA: Prentice-Hall, Inc., 1993.

[17] J. Yang, B. Geller, and S. Bay, "Bayesian and hybrid Cramer-Rao bounds for the carrier recovery under dynamic phase uncertain channels," IEEE Trans. on Signal Processing, vol. 59, no. 2, pp. 667-680, Feb 2011.

[18] S. Bay, B. Geller, A. Renaux, J. P. Barbot, and J. M. Brossier, "On the hybrid Cramer-Rao bound and its application to dynamical phase estimation," IEEE Signal Processing letters, vol. 15, pp. 453-456, 2008.

[19] S. Bay, C. Herzet, J. M. Brossier, J. P. Barbot, and B. Geller, "Analytic and asymptotic analysis of bayesian Cramer-Rao bound for dynamical phase offset estimation," IEEE Trans. on Signal Processing, vol. 56, no. 1, pp. 61-70, Jan. 2008.

[20] J. Yang and B. Geller, "Near-optimum low-complexity smoothing loops for dynamical phase estimation - Application to BPSK modulated signals," IEEE Trans. on Signal Processing, vol. 57, no. 9, pp. 37043711, Sep 2009.

[21] P. Kovintavewat and J. Barry, "Exit chart analysis for iterative timing recovery," vol. 4, pp. 2435-2439 Vol.4, Nov. 2004.

[22] S. Ten Brink, "Convergence behavior of iteratively decoded parallel concatenated codes," IEEE Trans. on Communications, vol. 49, no. 10 , pp. 1727-1737, Oct. 2001.

[23] N. Wu, H. Wang, J. Kuang, and C. Yan, "Performance analysis of codeaided symbol timing recovery on AWGN channels," IEEE Trans. on Communications, vol. 59, no. 7, pp. 1975-1984, July 2011.

[24] B. Mielczarek and A. Svensson, "Timing error recovery in turbo-coded systems on AWGN channels," IEEE Trans. on Communications, vol. 50, no. 10, pp. 1584-1592, Oct. 2002.

[25] H. Meyr, M. Moeneclaey, and S. A. Fechtel, Digital Communication Receivers, J. G. Proakis Series, Ed. Wiley Series in Telecommunications and Signal Processing, 1998.

[26] A. Masmoudi, F. Bellili, S. Affes, and A. Stephenne, "Closed-form expressions for the exact Cramer-Rao bounds of timing recovery estimators from BPSK, MSK and square-QAM transmissions," IEEE Trans. on Signal Processing, vol. 59, no. 6, pp. 2474-2484, Jun. 2011.

[27] M. Oerder, "Derivation of Gardner's timing-error detector from the maximum likelihood principle," IEEE Trans. on Communications, vol. 35, no. 6, pp. 684-685, 1987.

[28] J. G. Proakis, Digital Communications. McGraw-Hill, 1995.

[29] U. Mengali and A. N. D'Andrea, Synchronization Techniques for Digital Receivers. Plenum Press, New York and London, 1997.

[30] F. Gardner, Demodulator Reference Recovery Techniques Suited for Digital Implementation. Gardner Research Comp., 1988. [Online]. Available: http://books.google.fr/books?id=BqQ_NQAACAAJ

[31] W. C. Lindsey and M. K. Simon, Telecommunication Systems Engineering. Dover Publications, Incorporated, 1991.

[32] B. Vucetic and J. Yuan, Turbo Codes: Principles and Applications. Norwell, MA, USA: Kluwer Academic Publishers, 2000.

[33] N. Wu, "Performance analysis of code-aided iterative carrier phase recovery in turbo receivers," IET Communications, vol. 6, pp. 29802988(8), November 2012.

[34] L. Erup, F. M. Gardner, and R. A. Harris, "Interpolation in digital modems - part II: Implementation and performance," IEEE Trans. on Communications, vol. 41, no. 6, Jun. 1993.

[35] B. Geller, V. Capellano, J. M. Brossier, A. Essebbar, and G. Jourdain, "Equalizer for video rate transmission in multipath underwater communications," IEEE Journal of Oceanic Engineering, vol. 21, no. 2, pp. 150-156, Apr. 1996.

[36] J. M. Brossier, P. O. Amblard, and B. Geller, "Self adaptive PLL for general QAM constellations," in Proc. of $11^{\text {th }}$ European Signal Processing Conference EUSIPCO, Toulouse, Sept. 2002, pp. 631-635.

[37] I. Gradshteyn and I. Ryzhik, Table of Integrals, Series and Products, A. Jeffrey and D. Zwillinger, Eds. Elsevier Academic Press, 2007. 


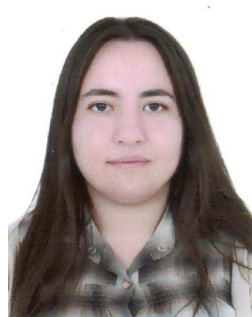

(WBAN).
Imen Nasr is currently working toward the Ph.D. degree in Information and Communications Technologies in both the Ecole Nationale Supérieure de Techniques Avancées (ENSTA) ParisTech - UPSA, France and the Ecole Supérieure des Communications de Tunis - Sup'Com (Higher School of Communication of Tunis), Tunisia. She received an Engineering and a Master Degree in Telecommunication from Sup'Com, University of Carthage, Tunisia, in 2011. Her research activities include synchronization and localization for Wireless Body Area Networks

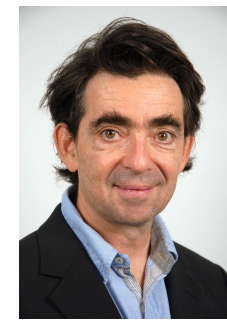

Benoit Geller (SM) received the Telecommunications engineering master degree from ENSEIRB and the $\mathrm{PhD}$ in Telecommunications from INP Grenoble in 1992. He was the head of the Multisensor and Information Team (TIM, 17 permanent researchers) at SATIE lab - ENS Cachan until he joined ENSTA ParisTech - UPSA in 2007 where he is currently a Full Professor. He works on wireless networks and the Internet of Things, and especially on iterative methods with application to digital communications. $\mathrm{He}$ has been involved in many European and industrial projects and has published about 100 international publications.

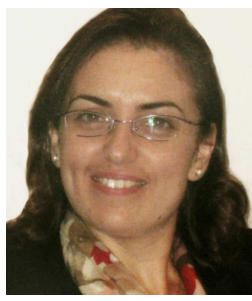

Leila Najjar Atallah received the engineering degree from Polytechnic School of Tunisia in 1997, a Master Degree in Automatic and Signal Processing from Ecole Supérieure d'Electricité Supélec, France in 1998, and a $\mathrm{PhD}$ in Sciences from University Paris XI in 2002. she joined the Higher School of Communications of Tunis (Sup'Com) as an Assistant Professor in 2006, where she is currently an Associate Professor. She carries her research activities in the research laboratory Communications, Signal and Image (COSIM) in Sup'Com. Her current research interests are in the field of statistical signal processing and in signal processing for wireless communications. They include channel estimation, synchronization and localization. She is also interested in sparse regularization problems with application to energy efficient Wireless Sensor Networks.

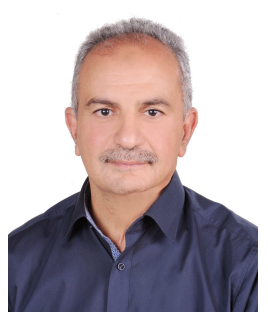

Sofiane Cherif is professor in telecommunication engineering at the Higher School of Communications of Tunis (SUP'COM), University of Carthage. He received engineering, MS degrees, andPh.D. in Electrical Engineering from the "National Engineering School of Tunis (ENIT)", University of Tunis-El Manar, in 1990 and 1998, respectively and the "Habilitation Universitaire" in Telecommunication from SUP'COM, in 2007. From 2011 to 2014, he was head of the doctoral school in ICT, and presently, he is the head of COSIM research Lab at SUP'COM. His current research interests are signal processing for communications, resource allocation and interference mitigation in wireless networks, wireless sensor networks and cognitive radio. 